\title{
Novel Frequency Swapping Technique for Conducted Electromagnetic Interference Suppression in Power Converter Applications
}

\author{
Ming-Tse Kuo ${ }^{1, *}$ and Ming-Chang Tsou ${ }^{2}$ \\ 1 Department of Electrical Engineering, National Taiwan University of Science and Technology, No. 43, \\ Section 4, Keelung Road, Da'an District, Taipei 106, Taiwan \\ 2 Leadtrend Technology Corporation, No. 1, Taiyuan 2nd St., Zhubei City, Hsinchu County 30288, Taiwan; \\ ming@leadtrend.com.tw \\ * Correspondence: mkuo@mail.ntust.edu.tw; Tel.: +886-2-2730-3288
}

Academic Editor: Ali M. Bazzi

Received: 9 August 2016; Accepted: 19 December 2016; Published: 27 December 2016

\begin{abstract}
Quasi-resonant flyback (QRF) converters have been widely applied as the main circuit topology in power converters because of their low cost and high efficiency. Conventional QRF converters tend to generate higher average conducted electromagnetic interference (EMI) in the low-frequency domain due to the switching noise generated by power switches, resulting in the fact they can exceed the EMI standards of the European Standard 55022 Class-B emission requirements. The presented paper develops a novel frequency swapping control method that spreads spectral energy to reduce the amplitude of sub-harmonics, thereby lowering average conducted EMI in the low-frequency domain. The proposed method is implemented in a control chip, which requires no extra circuit components and adds zero cost. The proposed control method is verified using a $24 \mathrm{~W}$ QRF converter. Experimental results reveals that conducted EMI has been reduced by approximately $13.24 \mathrm{~dB} \mu \mathrm{V}$ at $498 \mathrm{kHz}$ compared with a control method without the novel frequency swapping technique. Thus, the proposed method can effectively improve the flyback system to easily meet the CISPR 22/EN55022 standards.
\end{abstract}

Keywords: conduction electromagnetic interference (EMI); frequency swapping; frequency jittering; quasi-resonant flyback $(\mathrm{QRF})$

\section{Introduction}

Energy conservation and carbon reduction have received considerable public attention in modern society. Converters have been widely used in renewable energy generations, such as wind generation systems [1], photovoltaic systems and fuel cells [2]. To address such topics, this paper focused on the conversion efficiency of power supplies by investigating a high-efficiency quasi-resonant switching power supply [3-6]. According to the International Special Committee on Radio Interference (CISPR) 22 [7] or European Standard 55022 (EN55022) [7], as well as other relevant regulations, a power factor correction (PFC) mechanism must be installed in power converters with a rated power exceeding $75 \mathrm{~W}$. A PFC mechanism is necessary in devices such as television sets, network adapters, and notebook computer adapters. PFC generally uses a boost architecture to elevate the output voltage to reduce conduction loss. Therefore, a second-stage converter uses quasi-resonant switching power supply to reduce switching loss in switching devices. A quasi-resonant switching power supply operates the power devices at zero voltage switch (ZVS) and zero current switch (ZCS) conditions. Such switching techniques can create highly efficient products and can be widely applied in computer, communication, and consumer electronic products. Until now, no paper has explored the problems 
related to the electromagnetic interference (EMI) generated from the side-effect while the system efficiency is improved. In this paper, a novel frequency swapping technique is developed to meet CISPR 22/EN55022 standards without incurring additional component cost.

The power-supply switching devices generate very high current and voltage changes during the switching process, yielding high $\mathrm{d} I / \mathrm{d} t$ and $\mathrm{d} V / \mathrm{d} t$ and generating strong instantaneous dynamic noise when coupling parasitic inductance and parasitic capacitance [8]. Thus, electromagnetic noise generated in main circuit switching devices and relevant circuits is the main source of EMI in electronic devices. EMI primarily occurs as conduction and near-field interference sources. Certain high-frequency and high-power power supplies, such as high-frequency induction heating power supply and plasma power supply, can generate strong radiated EMI. The generation of EMI in power converters is depicted in Figure 1. Noise can be decomposed as common mode noise and differential mode noise components, which are transmitted through parasitic capacitance.

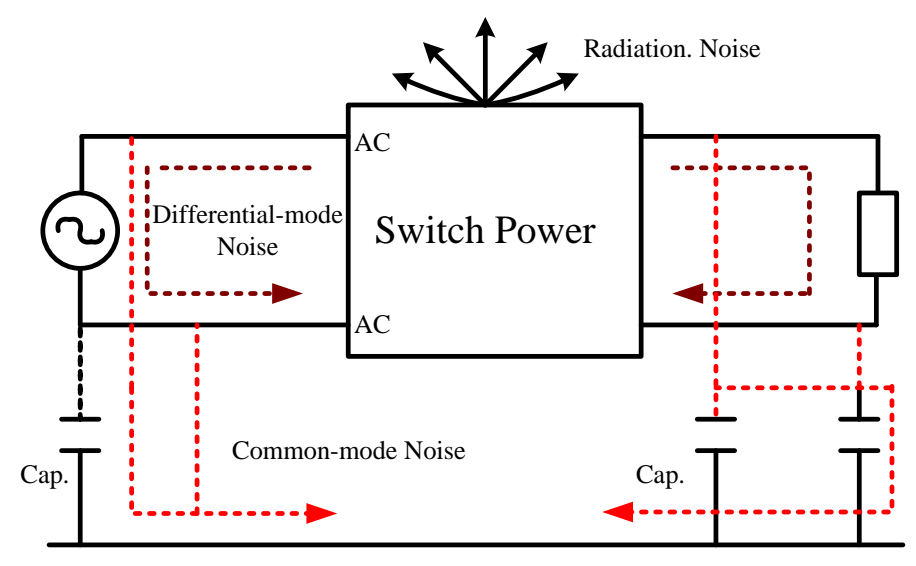

Figure 1. Electromagnetic interference (EMI) induced by a power converter.

High electromagnetic compatibility (EMC) is essential for electronic products. EMC design must be considered at the early stage of product design. Most converters operate with a fixed-frequency pulse-width modulation (PWM) control method. Most radiant energy is transmitted through a fundamental wave and sub-harmonic waves. Harmonic spectra generally have a narrow bandwidth and high amplitude. The amplitude of spectral components is the main item when analyzing EMI. Thus, EMI problems caused by fixed-frequency PWM are prominent. Frequency swapping is a technique that reduces the amplitude of harmonics by spreading spectral energy. In contrast to fixed-frequency PWM, frequency swapping refers to modulating the switching frequency around a fixed frequency during PWM, the principle of which is depicted in Figure 2a. When the signal periods in the time domain differ, spectra in the frequency domain spread with the decline in spectral amplitude, as shown in Figure $2 \mathrm{~b}$. The frequency swapping suppresses EMI at the source. It is an active suppression measure that inhibits both conducted EMI and radiant EMI [9].

Based on the spread spectrum principle, pulse frequency modulation (PFM) satisfies the feature of small harmonic amplitudes. However, when the commonly-used PFM control is adopted, the switching frequency is consistent with changes in load or input voltage (current). When the input voltage and load are fixed, PFM becomes another type of fixed-frequency PWM control, in which the frequency is not modulated [10]; thus, the advantage of PFM is lost. By contrast, frequency swapping techniques are unaffected by the output load and voltage. Switching frequency changes are controlled only by modulation signals and can thus easily attain the advantage of low EMI. In addition, when PWM is used for quasi-resonant converters, the voltage-second balance makes it impossible to achieve PWM control with fixed frequency. Instead, the operating frequency in a quasi-resonant system is mainly dominated by the magnetizing inductance. The prior swapping methods are only for fixed-frequency PWM system, and thus this paper provides a novel swapping 
control for quasi-resonant systems. As the frequency definitely varies, the complexity of the design is not increased by including designs for the magnetic components and the control loop in the system. Besides, how to realize frequency modulation is dominated by controllers, and it is feasible for aforementioned methods to be embedded in a chip. Actually, kinds of control integrated circuits (ICs) inclusive of above functions are available nowadays.

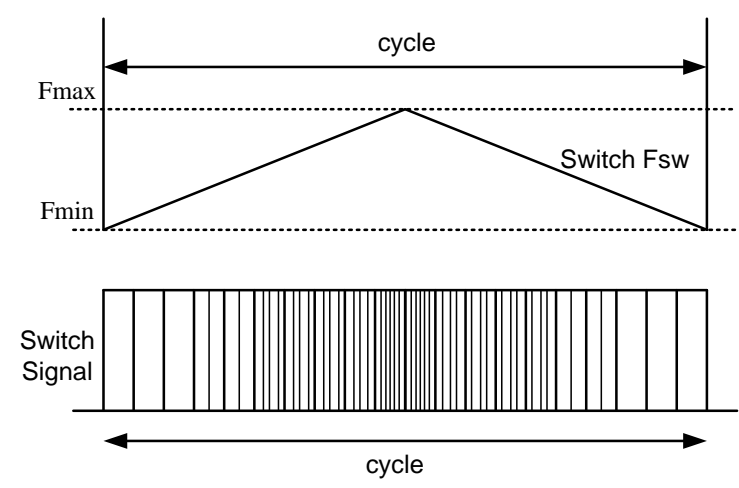

(a)

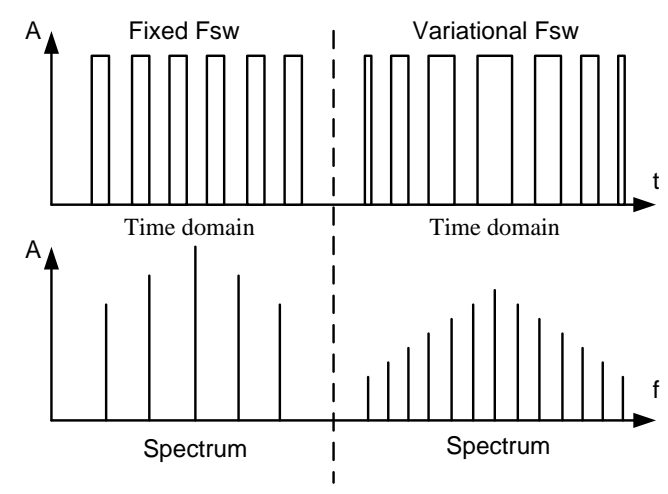

(b)

Figure 2. Frequency swapping technique and EMI suppression principle: (a) principle of switching frequency swapping; (b) fixed-frequency and variable-frequency signal spectra.

In this paper, a literature review is first presented, followed by an introduction of the frequency modulation (FM) principles, and its application in PWM carrier. The spectral characteristics of PWM waves are analyzed in detail. Second, the harmonic spectra generated by periodically-modulated PWM waveforms are analyzed; the control logic with the function of frequency swapping is implemented in an IC controller to verify the feasibilities.

In this paper, a $24 \mathrm{~W}(12 \mathrm{~V} / 2 \mathrm{~A})$ quasi-resonant flyback (QRF) converter is fabricated. The novel method is adopted to implement frequency swapping. A comparison of switching device voltage and current spectra of fixed-frequency and variable-frequency during operation shows that the amplitude of current and voltage harmonics during frequency swapping yields more favorable performance than that during fixed-frequency control in the low-frequency range. The proposed frequency swapping method reduces harmonic amplitudes by $10.2 \mathrm{~dB}$ at $646 \mathrm{kHz}$, proving the amplitude improvement and EMI suppression effects.

\section{Literature Review}

Currently, relevant studies can be roughly classified into experimental and commercial product design studies.

\subsection{Experimental Study}

Because frequency swapping modulates signals, related studies have typically emphasized periodically modulating switching frequencies. Workers at Polytechnic University of Catalonia [11] focused on selecting swapping modulation waveforms and implementing digital techniques. In addition, this research provided modulation waveform algorithms and the corresponding spectra, elaborated theoretical calculation methods, and briefly introduced EMC terminology and measurement instruments. However, the hardware circuit was at a verification stage because an expensive digital function generator was used for the functional circuit. In [12], the application of a frequency swapping technique for selecting rectifier modulation waveforms was discussed; however, no modulation circuit was provided. In [13], a complex theoretical method for determining spectral distribution equations was proposed. In [14], the influence of the resolution bandwidth of measurement instruments on the measurement results of modulated spectra was investigated, and the effect of filters on test results was 
summarized. The resolution bandwidth affects frequency offset and modulation frequency selection. However, the selection of the frequency swapping range and the inhibition of harmonic amplitude needs a trade-off. Hence, the specific value is difficult to define. In [14], the functional variation problem induced by periodic modulation in the original converter indicated that the output voltage ripple under an open loop condition is worse than that in a fixed-frequency PWM control system. Thus, a boost converter was used as a prototype to verify the argument. By contrast, the output voltage ripple in a close-loop buck converter with periodic modulation switching frequencies indicated suppression (yet incomplete removal) of low-frequency ripples. In [14], it was claimed without explanation that FM contributed to lowering the PFC circuit efficiency by $2.7 \%$ compared with that under fixed-frequency operation. The effect of FM on the original converter requires further investigation; the following researches are devoted to improve conduction EMI by choosing appropriate devices materials and optimizing those interconnections on PCB layout. In [15], Grounding schemes, material comparison between ferrite and nanocrystalline cores, and the new integrated filter structure are presented. The integrated structure maximizes the core window area and increases the leakage inductance by integrating both common-mode (CM) and differential-mode (DM) inductances onto one core and to reduce both DM and CM noise using a passive filter in a dc-fed motor drive. In [16], the proposed technique is a rule-based automatic procedure based on suitable databases that consider datasheet information of commercially available passive components (e.g. magnetic cores, capacitors). It allows the minimization of the filter's volume and therefore the improvement of the converter's power density. The size and the performance of EMI filters designed by the proposed procedure have been compared with those of a conventionally designed one. In [17], the proposed technique allows the CM and DM sections of the EMI filter to be properly selected in a more economical way, i.e., without the need of a dedicated hardware or costly radio frequency (RF) instrumentation. The filter has been set up according to a high power-density concept by using a high permeability nanocrystalline magnetic material for the CM choke core that allows a volume and weight reduction. In [18], a new approach for easy and fast modeling of EMI filter in aircraft application is proposed, in order to be used in an optimization process.

The following researches focused on exploring PWM-based random switching modulation techniques and chaotic switching modulation techniques [19]; these techniques have demonstrated satisfactory performance in reducing EMI amplitude and got considerable progress in this regard. However, the harmonic energy spread during modulation became too wide, thus deteriorating the low-frequency characteristics; moreover, it still suffers from uncontrollable energy spread and is inapplicable on QRF converters. Generating random and chaotic signals requires complex circuit structures, which increases the cost of fabrication. Thus, for the frequency swapping modulation method, the presented study applies a simple adjustable periodic modulation method to a QRF converter.

\subsection{Commercial Product}

In recent years, many corporations have developed power modules or control chips featuring a frequency swapping function. For example, following the single-chip switching power supply IC of the TinySwitch family of Power Integrations (San Jose, CA, USA), the TOP242, a dedicated switching power supply chip, was developed using the frequency swapping technique. The switching frequency varies in the range of $128-136 \mathrm{kHz}$, with a swapping range of $\pm 4 \mathrm{kHz}$ [20]. The product parameters are renewed constantly.

The spread oscillators LTC3809 and LTC6902 (Linear Technology, Milpitas, CA, USA) feature the functions of spread FM and pseudo-random noise techniques. The oscillators can generate any frequency between $5 \mathrm{kHz}$ and $20 \mathrm{MHz}$ [21]. In addition, the single-chip switching power controller NCP1215A (ON Semiconductor, Phoenix, AZ, USA) [22], ICE3B0365J (Infineon Technologies, Neubiberg, Germany), which has a $67 \pm 2.7 \mathrm{kHz}$ oscillation frequency [23], and FSDH0265 (Fairchild 
Semiconductor, Sunnyvale, CA, USA) [24] feature similar functions. However, no products applicable to quasi-resonant structures flyback converters are available.

All the aforementioned studies have explored frequency swapping in fixed-frequency controllers. Such an approach cannot be applied in QRF converters because when the system is operated in discontinuous conduction mode or boundary conduction mode, the system operating frequency depends on inductor volt-second balance. This paper proposes a novel frequency swapping control technique to solve these problems in variable FM systems.

\section{Frequency Modulation of Pulse-Width Module Signals}

This section involves the analysis of single-frequency sinusoidal modulation. The voltage waveform of converter switching devices resembles a rectangular pulse. The line input current is normally a sawtooth waveform. During operation, the current and voltage in a power device become increasingly complex. A simple analysis of sinusoidal modulation in PWM actuating signals is described as follows to predict the voltage characteristics at both ends of power devices.

\subsection{Single-Frequency Modulation of Pulse-Width Module Signals}

If $K\left(\omega_{c}\right)$ represents the switching function of an angular frequency $\omega_{c}$, then the unmodulated rectangular wave (PWM wave) signal can be expressed as:

$$
u_{C}(t)=U_{c m} \times K\left(\omega_{c} t\right)
$$

Thus, single-frequency sinusoidal modulation of a rectangular wave can be expressed as:

$$
U_{f m}(t)=U_{c m} \times K\left(\omega_{c} t\right)
$$

The Fourier series of rectangular waves with a duty cycle $D$ is expressed as:

$$
u_{C}(t)=U_{c m}\left\{D+\sum_{n=0}^{\infty} \frac{2}{n \pi} \sin (D n \pi) \cos \left(n \omega_{c} \tau\right)\right\}
$$

Let $\tau=t+\frac{m_{f}}{\omega_{c}} \sin \Omega t$, which can be substituted into (3) To obtain a Fourier series algorithm of FM rectangular waves:

$$
u_{f m}(t)=U_{c m}\left\{D+\sum_{n=0}^{\infty} \frac{2}{n \pi} \sin (D n \pi) \cos \left[n\left(\omega_{c} t+m_{f} \sin \Omega t\right)\right]\right\}
$$

Expanded from (4), (5) can be obtained:

$$
\begin{aligned}
u_{f m}(t)=U_{c m} D & +\frac{2}{\pi} U_{c m} \sin (D \pi) \cos \left(\omega_{c} t+m_{f} \sin \Omega t\right) \\
& +\frac{1}{\pi} U_{c m} \sin (2 D \pi) \cos \left(2 \omega_{c} t+2 m_{f} \sin \Omega t\right) \\
& +\frac{2}{3 \pi} U_{c m} \sin (3 D \pi) \cos \left(3 \omega_{c} t+3 m_{f} \sin \Omega t\right)+\cdots
\end{aligned}
$$

According to the previous section, FM of square waves is similar to FM of each sub-harmonic wave; however, the modulation index of each sub-harmonic wave in the modulated original carriers increases with the order of sub-harmonic waves. The modulation index of $n$th sub-harmonic waves is expressed as $m_{f(n)}=n \cdot m_{f}$, where $m_{f}$ represents the modulation index of carrier fundamental. Carson's rule is still applicable to the bandwidth of the boundary frequencies of each sub-harmonic wave, which can be expressed as:

$$
B W_{(n)} \approx 2\left(n \cdot m_{f}+1\right) \cdot f_{m}
$$


where $f_{m}$ is the frequency of the modulation waves. Generally, FM of non-sinusoidal waves is similar to FM of fundamental waves and each sub-harmonic wave. Because waveforms (or functions) that satisfy convergence conditions theoretically can be expressed as Fourier series expansion, the algorithm of FM current waveforms flowing through power devices can be obtained through similar approaches.

As shown in (6), the bandwidth of each modulated sub-harmonic wave increases with the harmonic order. If the sub-harmonic wave order is sufficiently high, nearby harmonic boundary frequencies will overlap as shown in Figures 3 and 4. Frequency overlap contributes to a uniform spectral distribution of high-order sub-harmonic waves; however, the amplitudes may increase because of component superposition (when the components are in the same phase) or attenuation (because of the reverse phase of nearby frequency components). However, from the perspective of energy diffusion, the amplitudes of spectral components at high frequencies during spectral superposition show an increasing tendency.

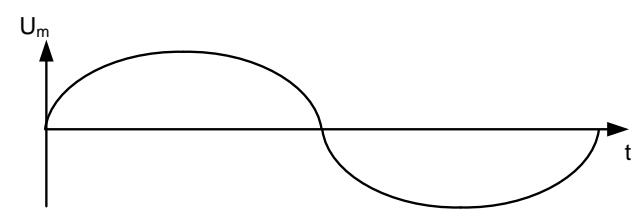

(a)

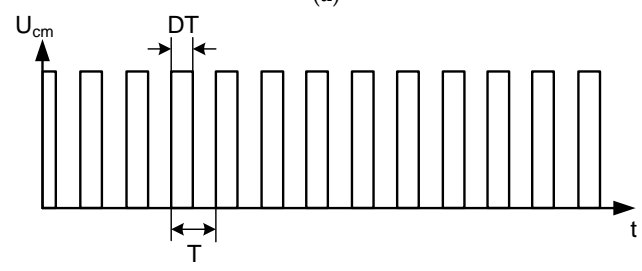

(b)

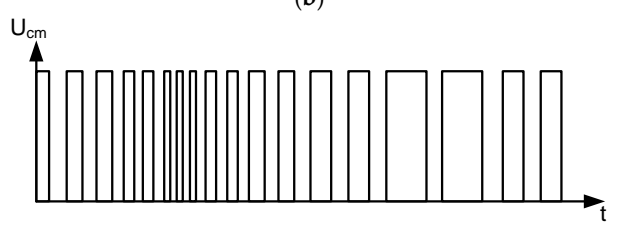

(c)

Figure 3. Sinusoidal modulation of FM rectangular waves: (a) Modulation Wave; (b) Carrier; and (c) PFM.

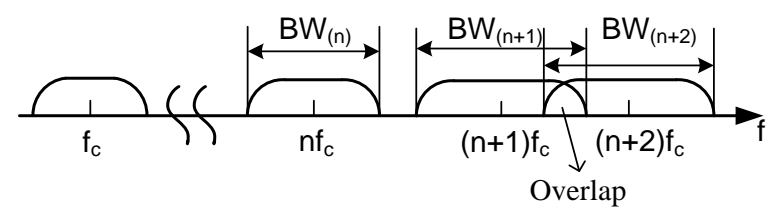

Figure 4. Sinusoidal modulation of FM square waves.

\subsection{Calculation of High-Order Sub-Harmonic Superposition}

Given that the sub-harmonic order is sufficiently high, the boundary components in the spectra superposition will increase the amplitudes in the affected area. Although the corresponding amplitudes are minimal in accordance with the increased harmonic order, potential interference at certain sensitive frequency bands is still unwanted at all [25].

Increases in amplitudes can be avoided in sensitive frequency bands by deriving the harmonic order during spectral superposition.

Accurate spectral distribution cannot be obtained easily in high-order sub-harmonic waves. Because the determined harmonic order is an integer, to facilitate calculation, the envelope of each sub-harmonic wave is hypothesized to be an ideal square frame [24], as depicted in Figure 5. 


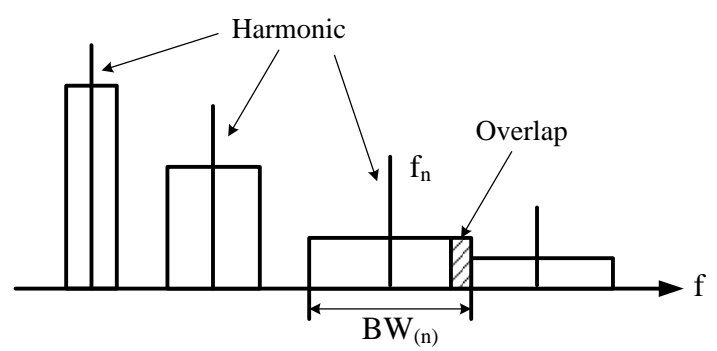

Figure 5. Boundary superposition of high-order sub-harmonic waves in modulated wave spectra.

The central frequency of each sub-harmonic wave of the original carrier is $f_{n}$, and the carrier fundamental frequency is $f_{c}$; thus, $f_{n}=n \cdot f_{c}$. Let the bandwidth of each sub-harmonic boundary of carriers be denoted as $B W_{(n)}$ and bandwidth of the fundamental wave be denoted as BW. Combining (6) and $m_{f}=\Delta f_{\text {cmax }} / f_{m}$ yields:

$$
B W_{(n)}=2\left(n \cdot m_{f} \cdot f_{m}+f_{m}\right)=2\left(n \cdot \Delta f_{c \max }+f_{m}\right)
$$

When the first superposition occurs, (8) is established for the superposition area:

$$
f_{n}+\frac{B W_{(n)}}{2}=f_{n+1}-\frac{B W_{(n+1)}}{2}
$$

where $n$ is the harmonic order.

Substituting (7) into (8) yields:

$$
n \cdot f_{c}+n \cdot \delta \cdot f_{c}+f_{m}=(n+1) f_{c}-(n+1) \cdot \delta \cdot f_{c}-f_{m}
$$

From (9), (10) is derived as follows:

$$
f_{c}=\frac{2}{1-\delta \cdot\left(1+2 \cdot n_{\text {overlap }}\right)} \cdot f_{m}
$$

where $f_{c}$ is the fundamental frequency of the carrier, $f_{m}$ is the modulated wave frequency, and $n_{\text {overlap }}$ is the carrier harmonic order during superposition. The $n_{\text {overlap }}$ is derived in (10) to obtain (11):

$$
n_{\text {overlap }}=\frac{1}{\delta} \cdot\left(\frac{1}{2}-\frac{f_{m}}{f_{c}}\right)-\frac{1}{2}
$$

According to (11), when the carrier fundamental wave frequency decreases, or when the modulation frequency $f_{m}$ and frequency offset $\delta$ increase, the harmonic order during superposition decreases. Generally, carrier fundamental wave frequencies are considerably higher than modulated wave frequencies (i.e., $f_{c}>f_{m}$ ). Thus, (11) can be approximately expressed as:

$$
n_{\text {overlap }}=\frac{1}{\delta} \cdot\left(\frac{1}{2}\right)-\frac{1}{2}
$$

In (12), considerable frequency offsets tend to generate spectral superposition in low harmonic waves. The trivial effect of modulated wave frequency $f_{m}$ on the superposition can be ignored.

Calculations after spectral superposition can be employed to predict the general distribution of high-order sub-harmonic boundaries. As stated previously, to prevent the potential hazard of high amplitudes in certain high-frequency bands, modulation frequency $f_{m}$ and frequency offset $\delta$ can be reasonably selected according to (12). Postponing spectral superposition until after the sensitive frequencies have been identified can avoid potential interference in the specific frequency bands. 
In reality, modulated waveforms cannot be single-frequency sinusoidal waves. When the modulated waveform contains only two modulated frequencies $\Omega 1$ and $\Omega 2$, which is already the simplest situation, the corresponding modulation indices will be $m f_{1}$ and $m f_{2}$, respectively. The analytic expression of a modulated wave is complex and the following components are included in the spectra:

(1) Carrier frequency $\omega_{\mathrm{c}}$ shows an amplitude proportional to $J_{0}\left(m f_{1}\right) J_{0}\left(m f_{2}\right)$.

(2) Boundary frequency $\left(\omega_{\mathrm{c}} \pm n_{\Omega 1}\right)$ shows an amplitude proportional to $J_{n}\left(m f_{1}\right) J_{0}\left(m f_{2}\right)$.

(3) Boundary frequency $\left(\omega_{\mathrm{c}} \pm n_{\Omega 2}\right)$ shows an amplitude proportional to $J_{0}\left(m f_{1}\right) J_{n}\left(m f_{2}\right)$.

(4) Additional boundary frequency (combined frequency; $\omega_{\mathrm{c}} \pm n_{\Omega 1} \pm n_{\Omega 2}$ ) shows an amplitude proportional to $J_{n}\left(m f_{1}\right) J_{0}\left(m f_{2}\right)$, where $\mathrm{n}$ and $\mathrm{p}$ are random integers.

Clearly, if the modulated waveform frequency is not a single frequency, then the generated spectral structure can be very complex. Thus, the present paper explores only the single-FM waveforms and corresponding spectra. Given that the modulated waveform is a single-frequency non-sinusoidal wave, it can be decomposed into a Fourier series. Because the frequency of each harmonic and the fundamental wave have an integer multiple relationship, $m f_{1}$ and $m f_{2}$ are proportional reasonably. Thus, these four types of boundary frequency components can be simplified. Nonetheless, spectrum analytic expression is intricate. In particular, when the modulated signal is discontinuous or presents irregular patterns, expressing it in analytic form is difficult. To address this, discrete methods can be employed to formulate a suitable equation.

In summary, this section explains the basic theory of frequency swapping and the principles of FM, and modulated PWM wave spectral characteristics are analyzed as well. Critical parameters related to FM theory are provided. In addition, the FM PWM wave spectral superposition at high-order sub-harmonic waves is explained and calculated carefully.

\section{Design of Frequency Swapping Circuits}

Figure 6 shows the close-loop control block diagram for a QRF converter. This is the generalized system shown above. The top part, $G_{0}(s)$ represents all the systems and all the controllers on the forward path. The bottom part, $G_{f}(s)$ represents all the feedback processing elements of the system. The letter " $G_{i}(s)$ " in the beginning of the system is called the Gain. We can define the Closed-Loop Transfer Function as follows:

$$
\frac{V_{O U T}(s)}{V_{I N}(s)}=\frac{G_{i}(s) G_{0}(s)}{1+G_{o}(s) G_{f}(s)}
$$

Perturbation feedback signals are added at the feedback end to modulate on-time of power switches. This new method doesn't affect the close-loop control system. The expected frequency swapping effect is obtained because the QRF converter is operated and based on ampere-second balance to reach ZCS.
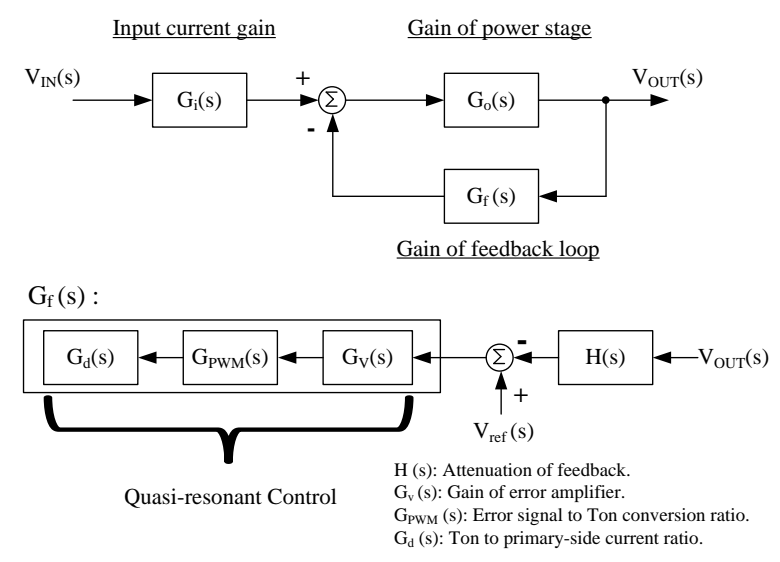

Figure 6. Control block diagram of the modulation waves. 


\subsection{Selection and Analysis of Frequency Swapping Techniques}

As indicated in Section 3, the amplitude, boundary bandwidth, and modulation indices of spectral components are closely related. Thus, modulation of the switching frequency proportionate to modulation of each sub-harmonic frequency can facilitate favorable EMI. The following sections explore the application methods. The methods and logic concepts share a common goal of adjusting the system operating frequencies to facilitate favorable EMI conduction. A simple analysis of the three methods are provided as follows.

\subsubsection{Change in Quasi-resonant Detection Delay Time}

As depicted in Figure 7, the quasi-resonant detection (QRD) mechanism detects whether the metal-oxide-semiconductor (MOS) drain to source (Vds) reaches the lowest point (valley). When the control IC detects a valley, the power switch is turned on to activate the valley switch and reduce the switching loss. Method 1 involves changing the interval between the QRD delay times (Points 1, 2, $3,4 \ldots$ repeat changes); subsequently, the system period and frequency can be adjusted. However, this method has two major disadvantages. First, the changeable period difference is only $\pm 300 \mathrm{~ns}$, yielding only small changes in the modulation index. Second, the QRF features enabling the activation of the valley switch while the power switch is on. When the QRD delay time is adjusted, switches in certain cycles cannot be activated at a valley time point, thereby lowering system efficiency, which can be worse than that of the original system. Thus, adjusting the QRD delay time is not applicable for the QRF structure.

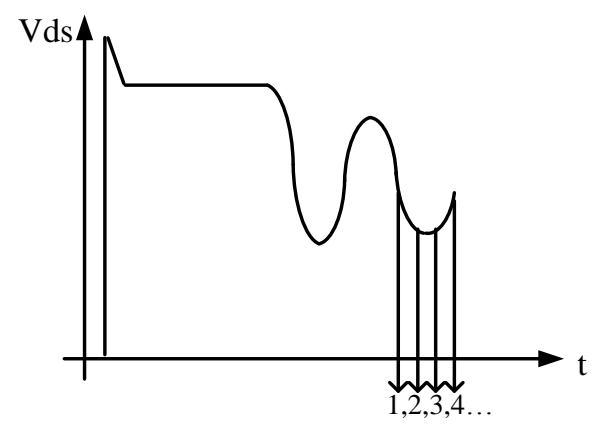

Figure 7. Change in quasi-resonant detection (QRD) delay signals.

\subsubsection{Change in Quasi-Resonant Flyback Valley Order}

Figure 8 shows the Vds waveform in the inductance and capacitance resonant state when the first-order power MOS and second-order diode in a flyback converter are switched off. Method 2 involves applying the control valley order to determine whether the power switch is to be switched on. In other words, in State 1, the third valley indicates that the power switch is to be switched on; in State 2, the second valley indicates that the power switch is to be switched on; and in State 3, the first valley indicates that the power switch is to be switched on. Subsequently, the state returns to State 1 and repeats continuously. Thus, the system period and frequency can be adjusted. However, this method has three major disadvantages. First, the changeable period difference is considerable, which easily causes a side effect of a large output ripple. Second, the resonant frequency relies on system inductance and MOS parasitic capacitance; modulation index variation between different systems cannot be controlled. Third, when the system is operated during the occurrence of the first valley, the control IC cannot change the valley switching order because of the volt-second balance principle. Thus, the method of adjusting the QRF valley order is not applicable to the QRF structure. 


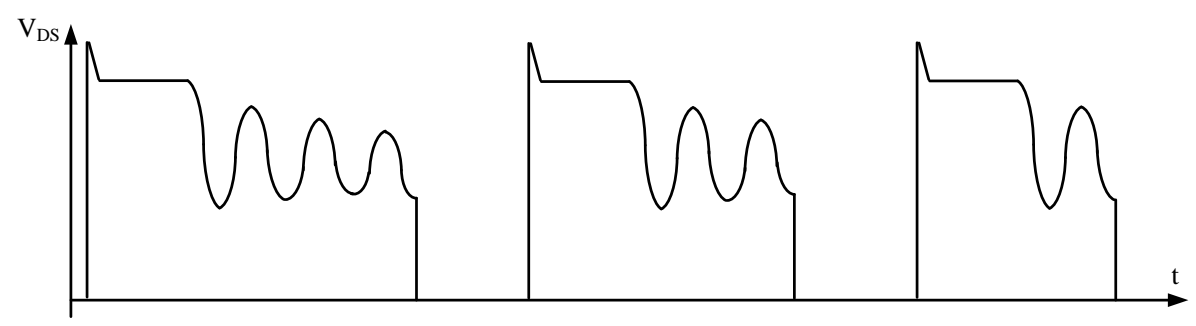

Figure 8. Valley order change.

\subsubsection{Disturbance on Voltage Feedback Signal}

As depicted in Figure 9, Method 3 causes direct disturbance on the voltage feedback signals. When the disturbance voltage is positive, the gate turn on $\left(T_{\text {on }}\right)$ increases and the gate turn off $\left(T_{\text {off }}\right)$ time increases, thereby enlarging the periods. By contrast, when the disturbance voltage is negative, the gate turn on $\left(\mathrm{T}_{\text {on }}\right)$ decreases and the gate turn off $\left(\mathrm{T}_{\text {off }}\right)$ time decreases, thereby changing the system periods and frequencies. This method has a disadvantage. Figure 10 defines the relative parameters of triangular modulation signals.

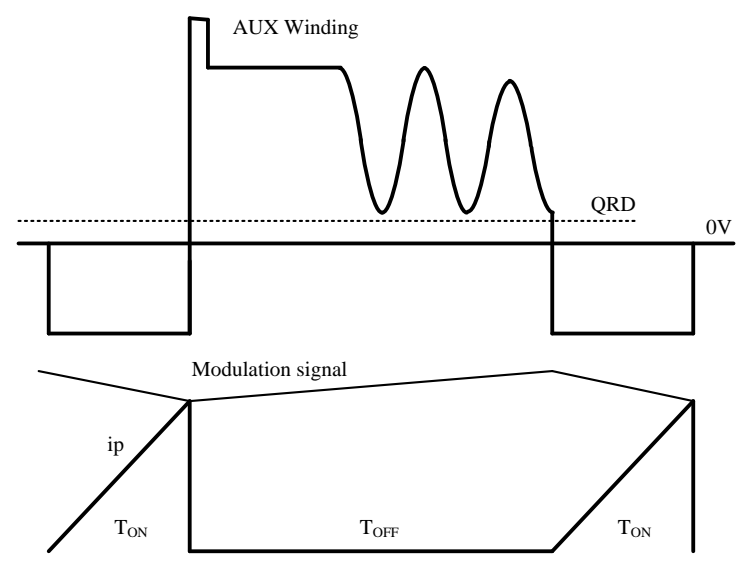

Figure 9. Disturbance voltage feedback signal.

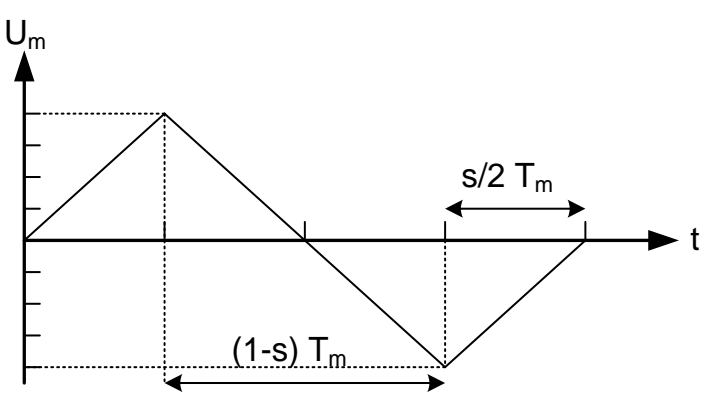

(a)

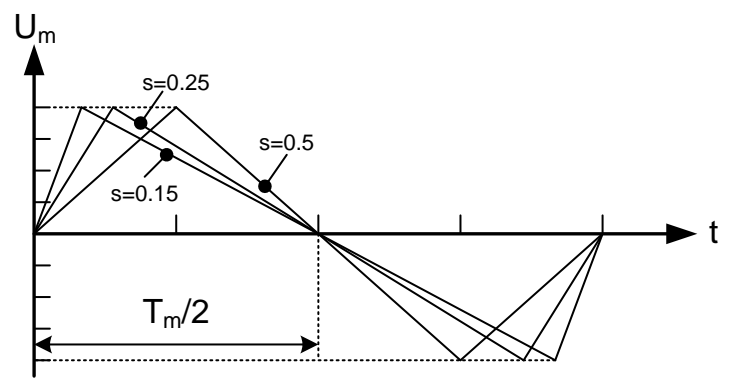

(b)

Figure 10. Triangular modulation signals: (a) signals; (b) different s ratio values.

The triangular wave expression is:

$$
u(t)\left\{\begin{array}{lr}
U_{m} \cdot f_{m} \cdot \frac{2}{s} t & 0 \leq t \leq s \cdot \frac{T_{m}}{2} \\
\frac{U_{m}}{1-s} \cdot\left(1-2 \cdot f_{m} \cdot t\right) & s \cdot \frac{T_{m}}{2} \leq t<(1-s / 2) \cdot T_{m} \\
\frac{2}{s} \cdot U_{m} \cdot\left(f_{m} \cdot t-1\right) & (1-s / 2) \cdot T_{m} \leq t<T_{m}
\end{array}\right.
$$


In Equation (14), $U_{m}, T_{m}$, and $f_{m}$ denote the amplitude, period, and frequency of triangular waveforms respectively. The $s$ parameter controls the location of the triangular peak between the original of the axis and the position of $T_{m} / 2$, named as peak parameter, and the range is restrained from 0 to 1 . The typical peak parameter is 0.5 commonly, which makes the triangular waveforms central-symmetrical and axis-symmetrical. The spectral composition of triangular modulation still meets the rules that the attenuation of bandwidth and amplitude increases when the modulation parameter increases. Compared with sinusoidal modulation, the spectral of triangular modulation is smoother. Next, the frequency of modulation waveforms needs to be defined. Because the frequency modulation spreads the system intrinsic frequency, parts of spread frequency enter the region lower than operating frequency of the system. Particularly, the spread frequency is forbidden to be lower than acoustic frequency. The acoustic noise often occurs at fundamental waves which own the lowest frequency among all harmonic waves. Hence, Equation (15) must be satisfied as:

$$
f_{c}-\frac{B W}{2}=f_{c}-\left(\Delta f_{c \max }+f_{m}\right)>f_{\text {amax }}
$$

In Equation (15), $f_{c}$ is the frequency of the carrier waveforms; $B W$ is the bandwidth; $f_{a m a x}$ is the maximum of acoustic frequency about $20 \mathrm{kHz}$. The switching frequencies of low-power converters are commonly located between hundreds of $\mathrm{kHz}$ and numbers of $\mathrm{MHz}$, which makes the acoustic frequency easier to be avoided. However, for converters with tens of $\mathrm{kHz}$ frequency, the condition of Equation (15) must be cared and observed. Therefore, the thesis uses the triangular modulation with $s$ parameter as 0.5 and modulation frequency as $1 \mathrm{kHz}$.

However, this method does not have the flaws of Method 1 or 2, nor is it confined by the system components. To achieve the optimal frequency swapping method and minimize induced problems on systems, the disturbance voltage feedback-signal method is the most suitable solution for application on the QRF structure. In the following exploration and analysis section, the disturbance voltage feedback signal structure is employed to design a converter.

\subsection{Embedded Logic Circuit of Adjustable Frequency Swapping}

Before the control IC is activated, the auxiliary winding of a transformer is considered as a route to ground. The resistance of copper coils of the auxiliary winding is measured as $106 \mathrm{~m} \Omega(50 \mathrm{~Hz})$ which is far smaller than $R A$ resistor in Figure 11. Thus, parallel resistors $\left(R_{\text {total }}=R A / / R B\right.$ in Figure 11$)$ can be applied to calculate the total external impedance from the FB pin in Figure 11. When VCC in Figure 12 encounters under-voltage-lockout (UVLO ON), the IC generates a current source of approximately $50 \mu \mathrm{A}$. As shown in Figures 11 and 12, this state enables the FB pin to determine the adjustable frequency swapping range. The adjustable range and setting values are listed in Table 1 . This adjustable frequency swapping range enables users to control the disturbance and optimize the output ripple standards.

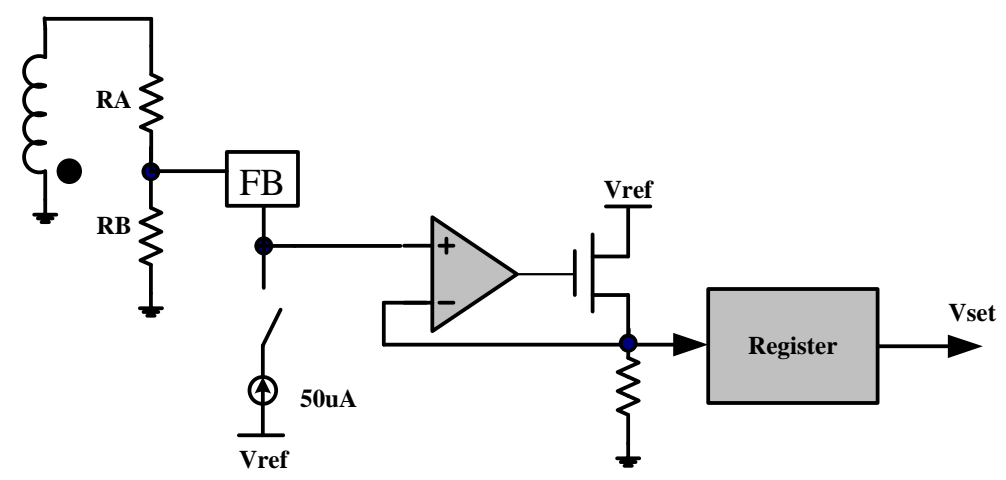

Figure 11. Circuit for adjusting the frequency swapping range. 


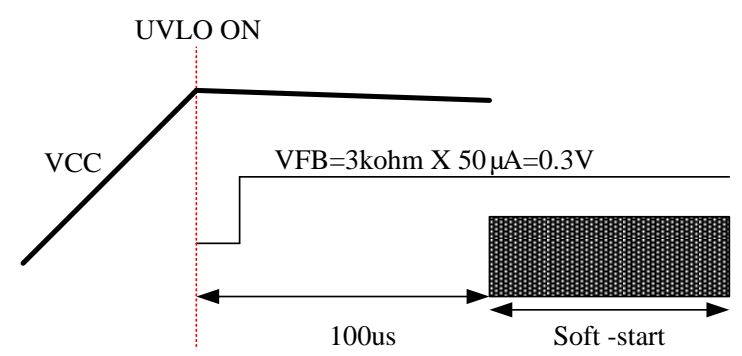

Figure 12. Embedded logic circuit for adjusting the frequency swapping range.

Table 1. Comparison of swapping range settings.

\begin{tabular}{cccc}
\hline Ideal $\boldsymbol{R}_{\text {total }}$ & $\boldsymbol{V}_{\boldsymbol{F B}}$ Range & Suggested $\boldsymbol{R}_{\text {total }}$ Value & Swapping Range (\%) \\
\hline $40 \mathrm{k}<R_{\text {total }}$ & $1.7<V_{F B}$ & $42 \mathrm{k}$ & \pm 9 \\
$22 \mathrm{k}<R_{\text {total }}<40 \mathrm{k}$ & $1.1<V_{F B}<1.7$ & $30 \mathrm{k}$ & \pm 6 \\
$14 \mathrm{k}<R_{\text {total }}<22 \mathrm{k}$ & $0.7<V_{F B}<1.1$ & $20 \mathrm{k}$ & \pm 3 \\
$R_{\text {total }}<14 \mathrm{k}$ & $V_{F B}<0.7$ & $10 \mathrm{k}$ & \pm 0 \\
\hline
\end{tabular}

\subsection{Embedded Logic Circuit of Feedback Disturbance}

Based on Figure 13 and Equation (16), after choosing adequate $R_{C}$ in Figure 13 and determining frequency swapping, this logic circuit will adjust $R_{D}$ to alter frequency swapping range, and the resistance of $\mathrm{R} 1$ is decided by an 8-bit binary code.

This switch array switches in 16 steps. The clock signal in Figure 13 is the FM disturbance period (in cycles) for adjusting the $\mathrm{R}_{\mathrm{C}}$ resistance.

$$
C_{\text {comp_ } J}=V_{\text {comp }} \times\left(1 \pm \frac{R_{C}}{R_{D}}\right)
$$

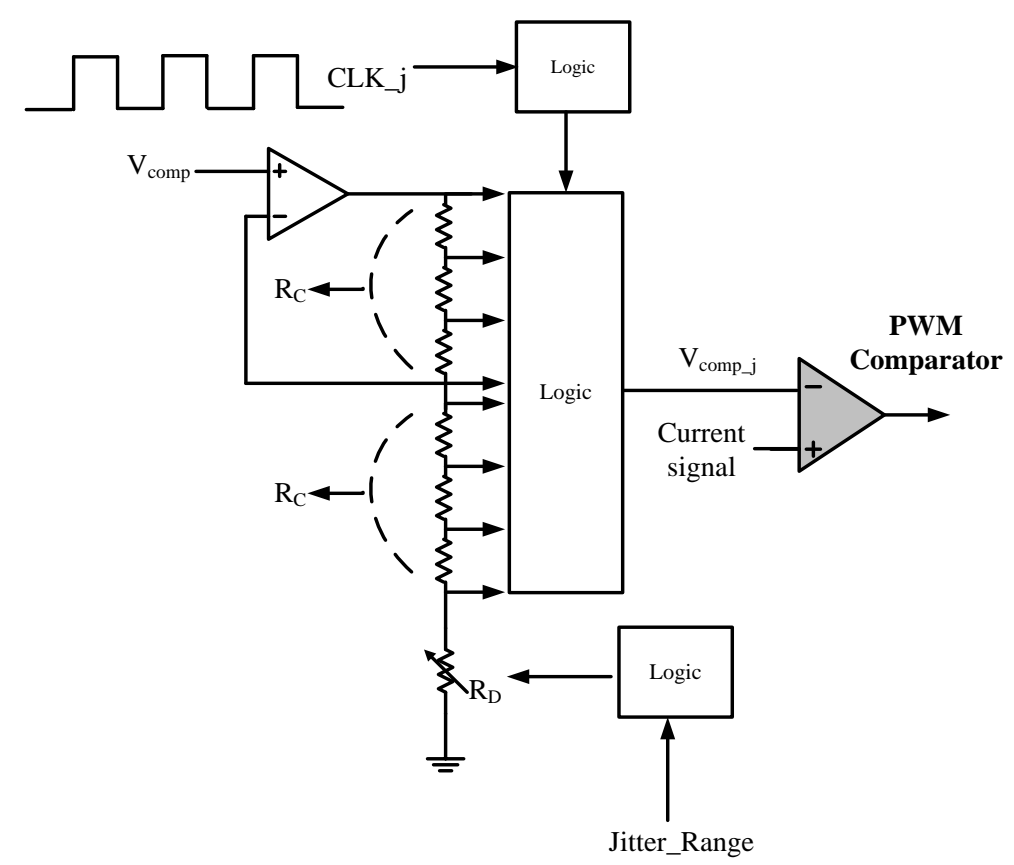

Figure 13. Frequency swapping logic circuit. 


\section{Results and Analysis}

The theoretical analysis validates the feasibility of the proposed technique and the results are compared in two parts. First, SIMPLIS simulation software (SIMPLIS Technologies, Inc., Portland, OR, USA) was used to verify and determine the optimal parameters. Second, a novel frequency swapping technique was incorporated into the control chip (Figure 14) to fabricate a practical prototypical converter for comparison (Figure 15).

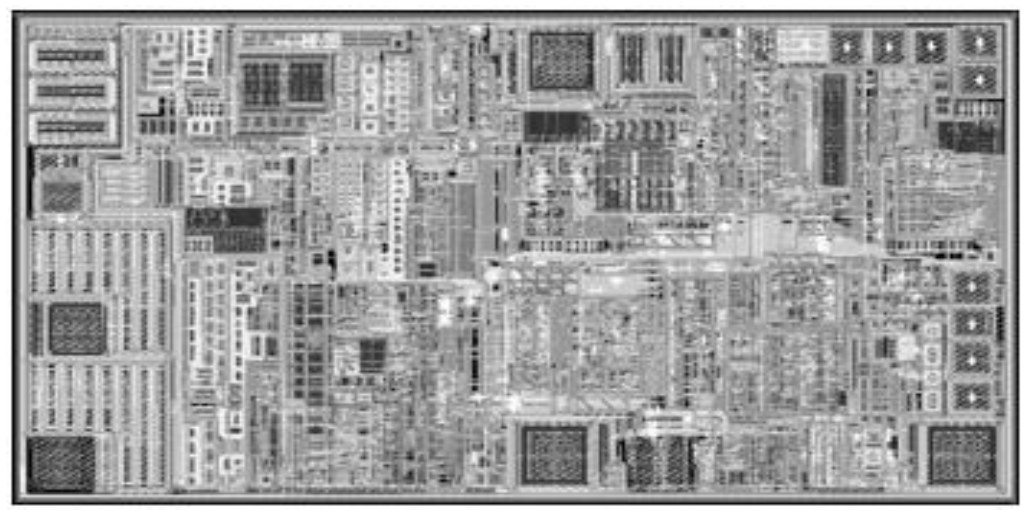

Figure 14. Integrated circuit (IC) layout of the control chip (Taiwan Semiconductor Manufacturing Company; $0.6 \mu \mathrm{m} 7 \mathrm{~V} / 12 \mathrm{~V}$ ) featuring the novel frequency swapping technique.

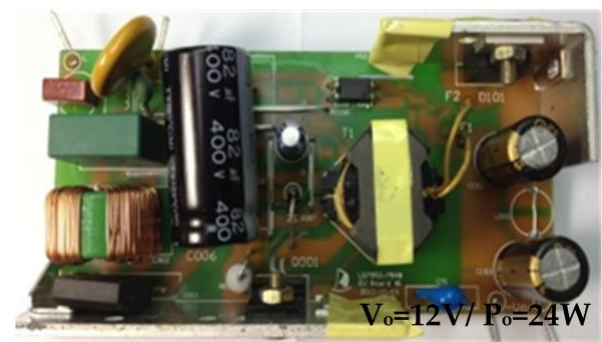

(a)

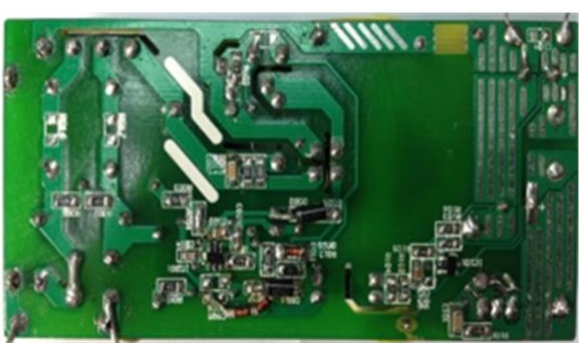

(b)

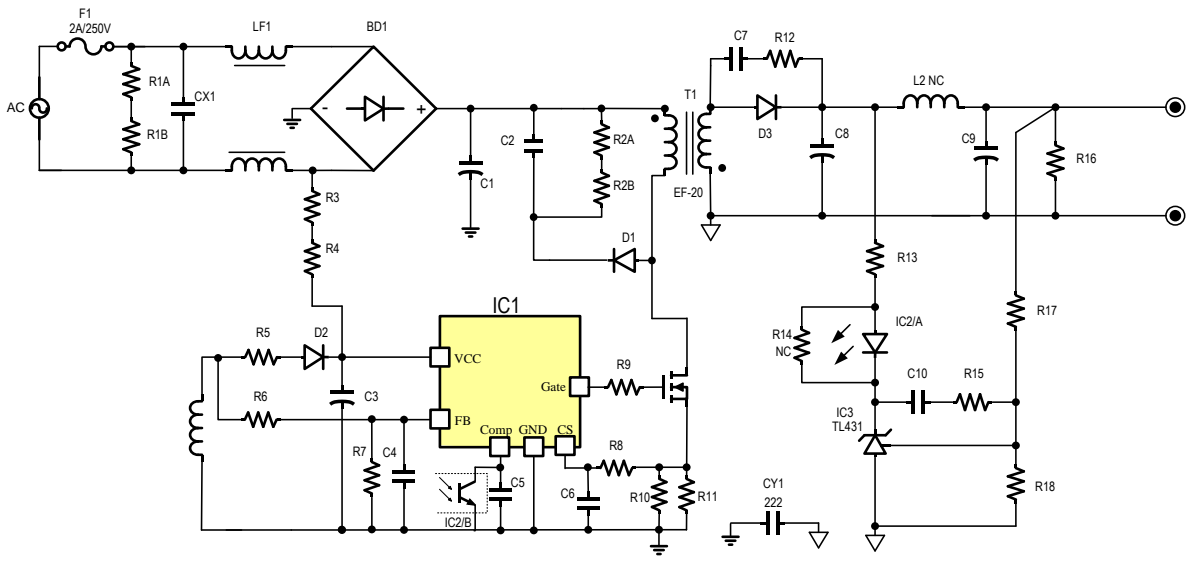

(c)

Figure 15. A photograph of the prototypical converter: (a) top layer; (b) bottom layer; and (c) detailed schematic of QRF.

The typical circuit of QRF converter is shown in Figure 15c. It mainly depends on a controller that provides necessary QRD (FB PIN) signals and voltage mode functions: under current mode control, this controller detects the inductance current through a current-sensing resistor (CS PIN) and catches output voltage signal through optical coupler to determine on time duty cycle. When $R 6$ and $R 7$ 
will divide the voltage signal into the FB pin by the auxiliary winding. Chip will be able to set the swapping range $\left(R_{\text {total }}=R 6 / / R 7\right)$ and determine the QRD signal. By the way, the circuit involves the CM choke (LF1) and filtering X-capacitor (CX1) to inhibit conduction EMI. In the following, our discussion will focus on these devices and analyze their pros and cons. Table 2 lists the external system component parameters.

Table 2. Hardware and specifications.

\begin{tabular}{cc}
\hline Hardware/Simulation Parameters & Specifications \\
\hline Input Voltage & $V_{\text {in }}=90 \mathrm{~V}-264 \mathrm{~V}$ \\
Output Voltage & $V_{o}=12 \mathrm{~V}$ \\
Output Current & $I_{o}=0-2 \mathrm{~A}$ \\
Switch Frequency & $f_{S}=65 \mathrm{kHz}$ \\
Max. Duty Cycle & $D_{\max }=0.48$ \\
Transformer & $L p=1.6 \mathrm{mH}$ \\
Ratio of Transformer & $N m=0.8$ \\
Output Capacitance & $C_{o}=680 \mathrm{uF} \times 2 ; E S R=0.01 \mathrm{ohm}$ \\
\hline
\end{tabular}

\subsection{Simulation Results}

At first, the functional circuit for frequency swapping block is built by SIMPLIS as shown in Figure 16a shows that the binary code for frequency swapping was used to control the $\mathrm{R}_{1}$ resistance and an eight-step variable-frequency periodic cycle was selected to make the operating frequency changing from 130 to $0.7 \mathrm{kHz}$ within a single step. By contrast, Figure $16 \mathrm{~b}$ shows how to control the R1 resistance through the switches. Through the binary signals (Qib and Qi), changes in the sink and source current are switched to generate positive and negative voltage differences from the disturbed voltage feedback, thereby achieving voltage compensation disturbance. Finally, Figure 16c shows how FM signals are generated by using a low-frequency triangular wave generator, and subsequently the cyclic frequency swapping repeats.
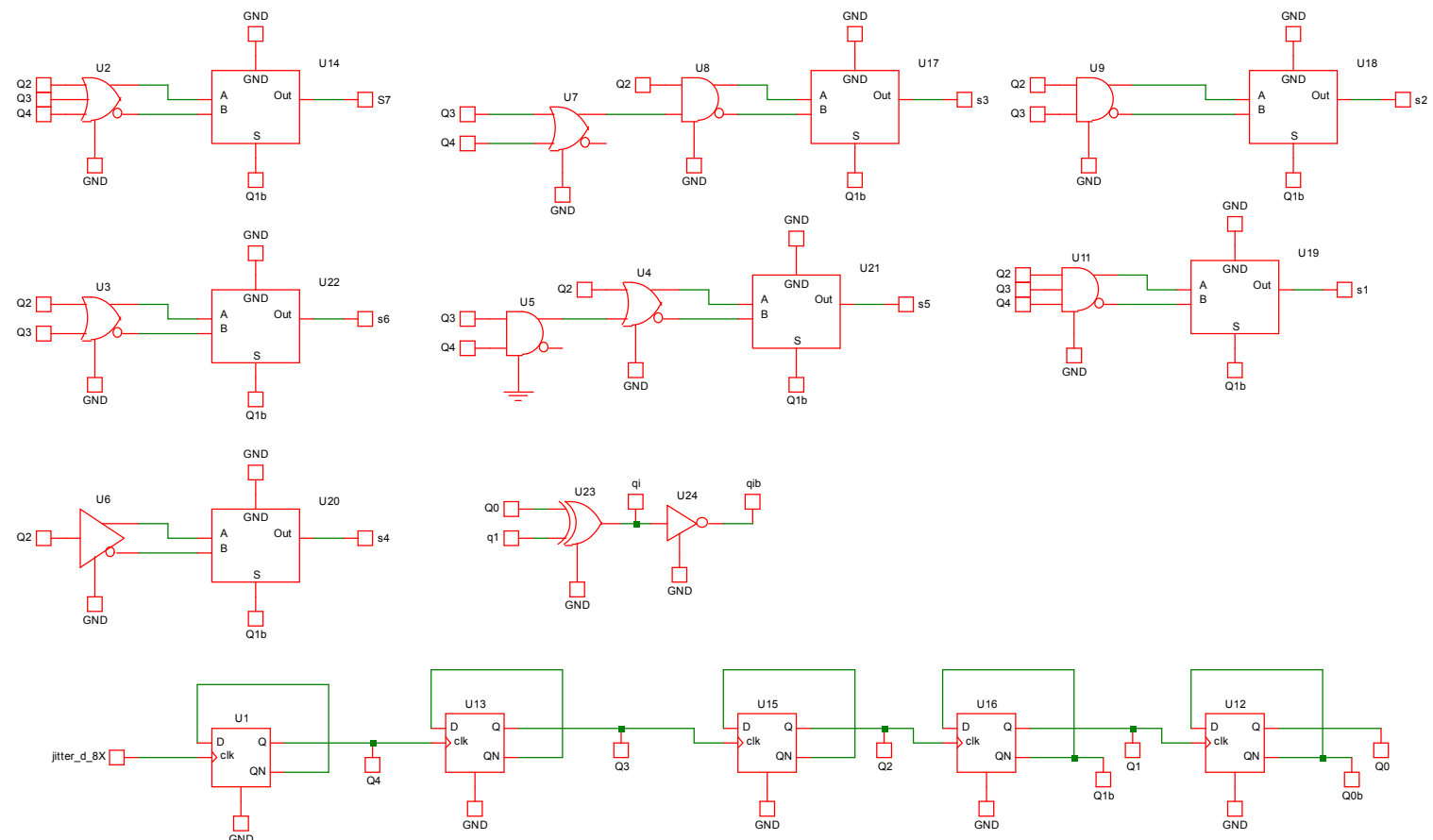

(a)

Figure 16. Cont. 


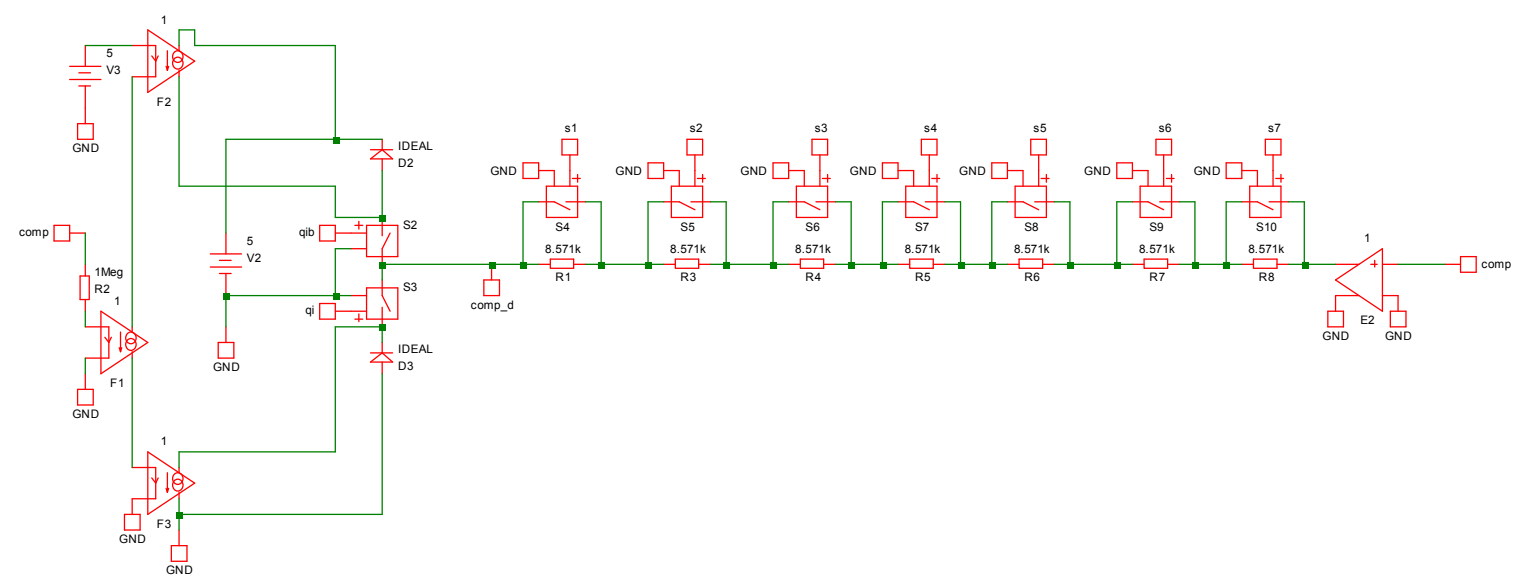

(b)

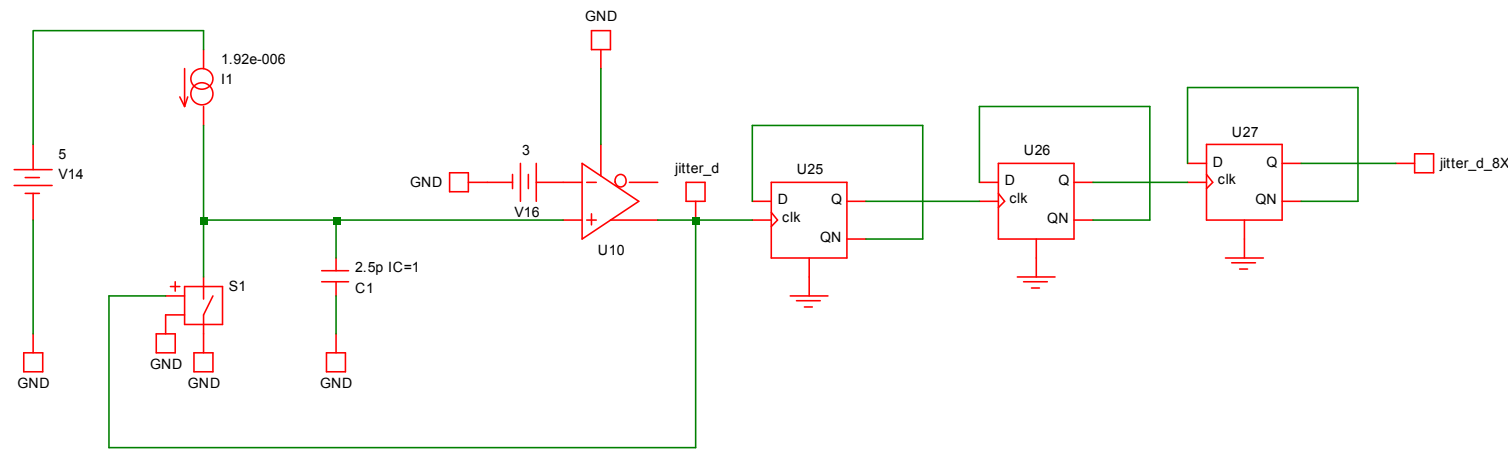

(c)

Figure 16. The novel frequency swapping circuit: (a) binary code for frequency swapping; (b) ratio for frequency swapping; and (c) frequency swapping modulation.

Figure 17 and Table 3 further indicate that larger frequency offsets $\left(\Delta F_{s w}\right)$ result in greater amplitude attenuation. However, the frequency offset is limited by the circuit design in actual converters.

(1) When the frequency offset is too high, although the duty cycle remains unchanged, the corresponding component conduction time decreases. The $\mathrm{T}_{\text {on }}$ and $\mathrm{T}_{\text {off }}$ time occupy a major portion of duty cycle, thus hindering energy transmission. When the switching frequency is too low, the corresponding conduction time increases, which probably results in magnetic component saturation.

(2) When the frequency offset is too high, energy transmission becomes uneven, which possibly induces large output ripples if the system cannot response this voltage feedback disturbance rapidly.

Although the simulation results cannot verify the statement in Passage (1), when $R_{\text {total }}=52 \mathrm{k} \Omega$ the $\Delta F_{s w}$ disturbance is within $\pm 12 \%$ (Table 3), the output ripple exceeds the regulated tolerance $(100 \mathrm{mV})$. Thus, in the following section, the maximum frequency swapping adjustment range is set as $\pm 9 \%$ in the IC design $\left(R_{\text {total }}=40 \mathrm{k} \Omega\right)$. In response to the modulation index, this paper sets the modulation index to $1 \mathrm{~ms}$, because the system voltage feedback mechanism can track and compensate the index, consequently mitigating the disturbance effect if the modulation index is less than $1 \mathrm{~ms}$. By contrast, when the modulation index is higher than $1 \mathrm{~ms}$, it easily enters the hearing range $(1-20 \mathrm{kHz})$, which forbids its application in computer, communication, and consumer electronic products. Thus, in the IC FM parameters, the modulation index is set at $1 \mathrm{~ms}$. 
Table 3. Comparison of modified swapping ratios.

\begin{tabular}{|c|c|c|c|c|c|}
\hline \multirow{2}{*}{$\begin{array}{l}X \text {-Capacitor } \\
\text { Value }\end{array}$} & \multirow{2}{*}{$\begin{array}{l}\text { Comparison of } \\
\text { Swapping } \\
\text { Range Settings }\end{array}$} & \multirow{2}{*}{$\begin{array}{c}\text { Frequency } \\
\text { Swapping } \\
\text { Ratio } \\
\end{array}$} & \multirow{2}{*}{$\begin{array}{c}\text { Frequency } \\
\text { Swapping } \\
\text { Period }\end{array}$} & \multicolumn{2}{|c|}{ Output Ripple } \\
\hline & & & & $90 \mathrm{~V} / 60 \mathrm{~Hz}$ & $264 \mathrm{~V} / 50 \mathrm{~Hz}$ \\
\hline \multirow{5}{*}{$0.22 \mu \mathrm{F}$} & $8.61 \mathrm{k} \Omega$ & $\pm 0 \%$ & $1 \mathrm{~ms}$ & $\Delta 3 \mathrm{mV}$ & $\Delta 5.2 \mathrm{mV}$ \\
\hline & $18.18 \mathrm{k} \Omega$ & $\pm 3 \%$ & $1 \mathrm{~ms}$ & $\Delta 21 \mathrm{mV}$ & $\Delta 41 \mathrm{mV}$ \\
\hline & $29 \mathrm{k} \Omega$ & $\pm 6 \%$ & $1 \mathrm{~ms}$ & $\Delta 32 \mathrm{mV}$ & $\Delta 71 \mathrm{mV}$ \\
\hline & $40 \mathrm{k} \Omega$ & $\pm 9 \%$ & $1 \mathrm{~ms}$ & $\Delta 48 \mathrm{mV}$ & $\Delta 98 \mathrm{mV}$ \\
\hline & $52 \mathrm{k} \Omega$ & $\pm 12 \%$ & $1 \mathrm{~ms}$ & $\Delta 102 \mathrm{mV}$ & $\Delta 143 \mathrm{mV}$ \\
\hline
\end{tabular}

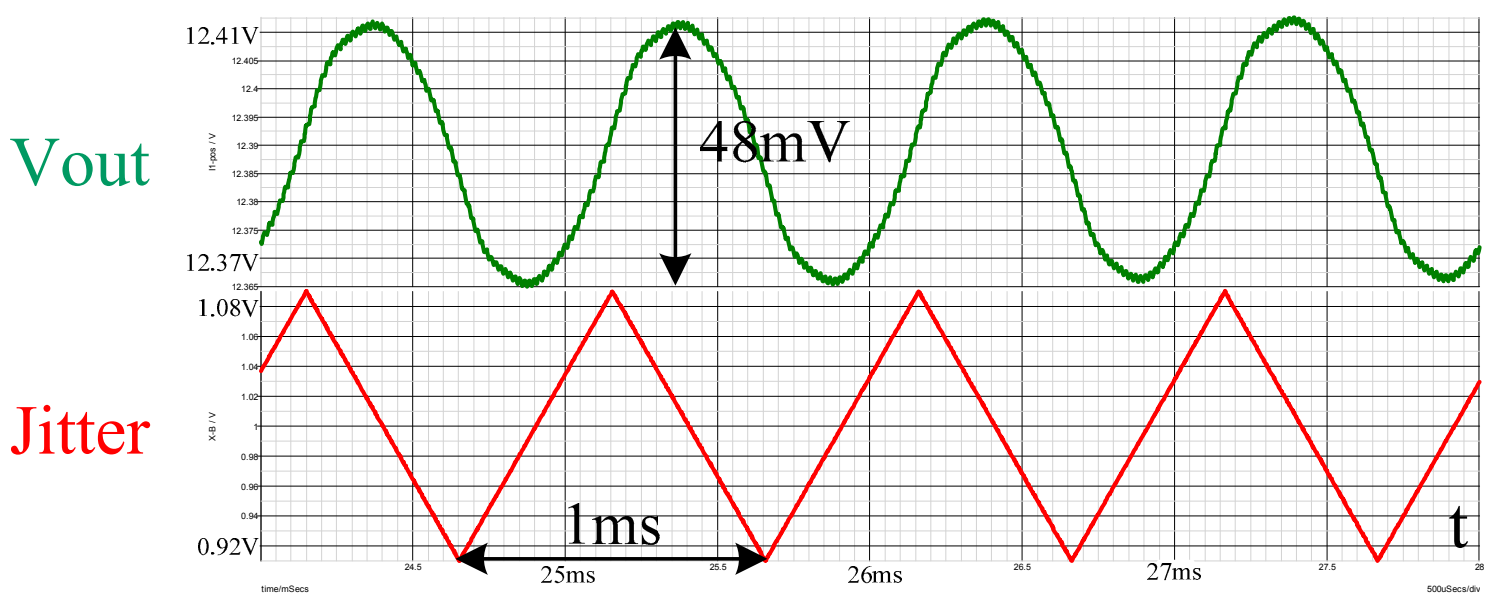

(a)

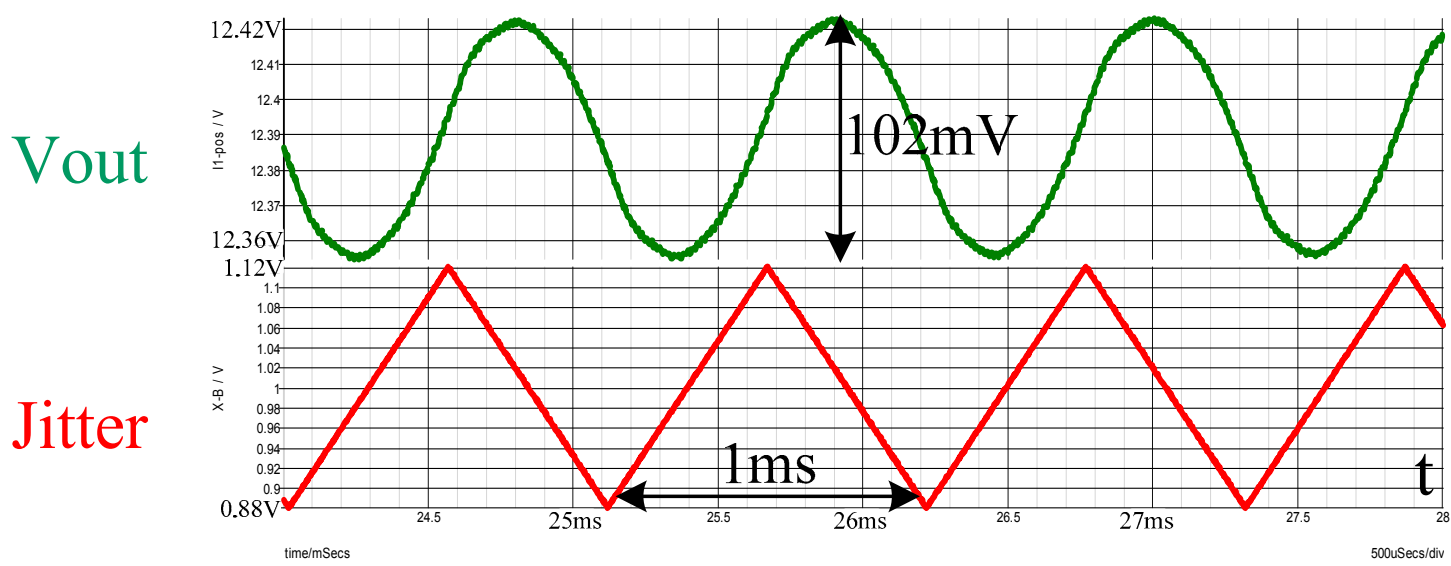

(b)

Figure 17. Modified frequency swapping ratio comparison: (a) output ripple for $\pm 9 \%$ frequency swapping range $(90 \mathrm{~V} / 60 \mathrm{~Hz})$; (b) output ripple for $\pm 12 \%$ frequency swapping range $(90 \mathrm{~V} / 60 \mathrm{~Hz})$.

\subsection{Waveform Measurement}

As shown in Figure 18, the system sets impedance from the FB pin to adjust the frequency swapping range as $R_{\text {total }}=40 \mathrm{k} \Omega, 29 \mathrm{k} \Omega, 18.18 \mathrm{k} \Omega$, and $8.61 \mathrm{k} \Omega$ to achieve the expected frequency swapping range. 


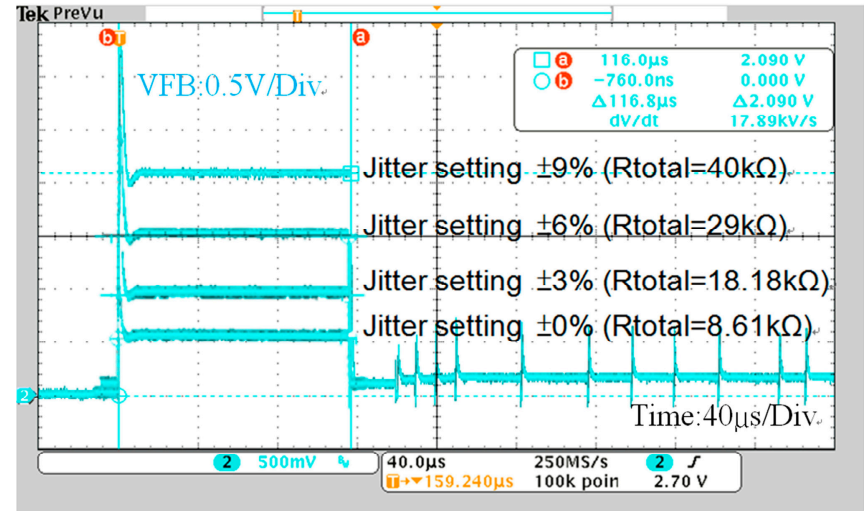

Figure 18. Using the feedback (FB) pin to set frequency swapping range.

The spectral analysis function of the oscilloscope is used to intercept the time domain waveform of the PWM wave at a fixed frequency swapping modulation and the spectrum within the frequency swapping range (Figure 19). Figure 19a shows there is no frequency amplitude for a system working frequency of $45.6 \mathrm{kHz}$; thus, this setting is used for the control group. Figure 19b shows that for a system working frequency of $45.6 \pm 1.45 \mathrm{kHz}$, the frequency swapping amplitude is $\pm 3 \%$. Figure $19 \mathrm{c}$ shows that for a system working frequency of $45.6 \pm 2.9 \mathrm{kHz}$, the frequency swapping amplitude is $\pm 6 \%$. Figure $19 \mathrm{~d}$ shows that for a system operating frequency of $45.6 \pm 4.4 \mathrm{kHz}$ means the maximum amplitude of the frequency swapping of $\pm 9 \%$; therefore, this frequency swapping range $( \pm 4.4 \mathrm{kHz})$ is adopted for the comparison group in this paper. According to the aforementioned measurement data, the FM parameters and frequency swapping range are identical with simulated conditions.

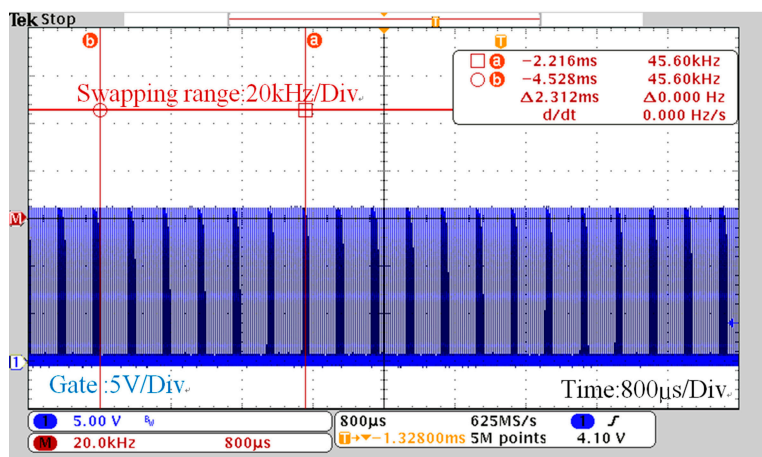

(a)

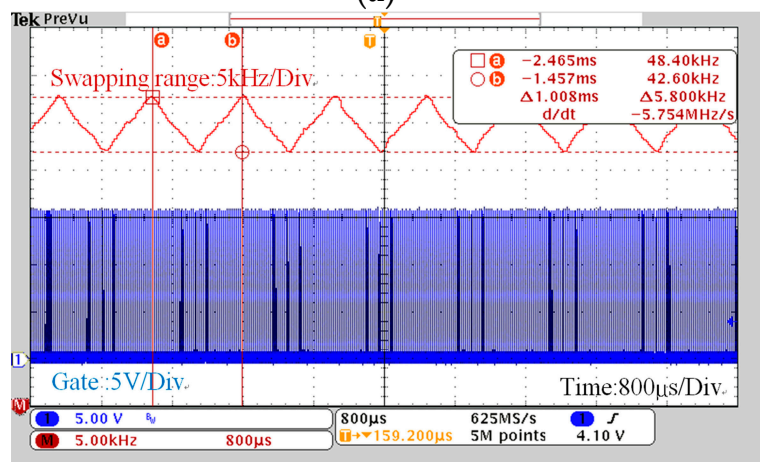

(c)

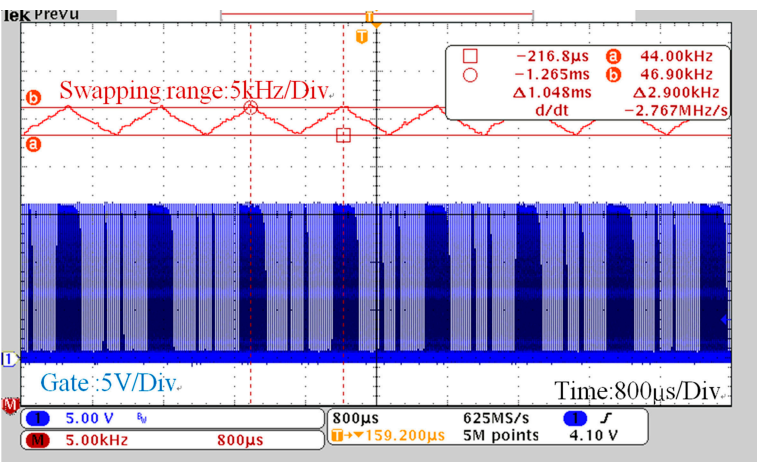

(b)

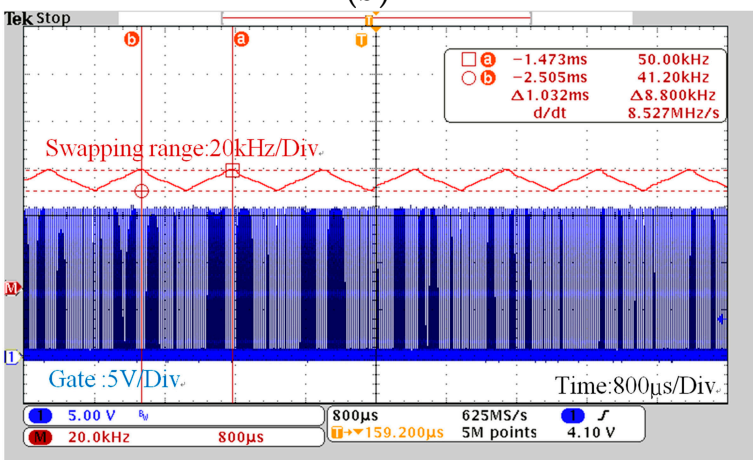

(d)

Figure 19. Measurement of operating frequencies. (a) no frequency swapping; (b) frequency swapping range set at $\pm 3 \%$; (c) frequency swapping range set at $\pm 6 \%$; and (d) frequency swapping range set at $\pm 9 \%$. 


\subsection{Testing Conducted Electromagnetic Interference}

The $24 \mathrm{~W}$ prototypical converter used in this thesis is applied to high resistive load, which commonly uses $\pi$ filter to filter high-frequency noise. Based on the frequency of the unwanted noise to be inhibited, the crossover frequency and the components (common mode choke and X-capacitor) of the filter can be designed to improve the noise immunity. To test the conducted EMI is divided into two parts at high input voltage $(230 \mathrm{~V} / 50 \mathrm{~Hz})$.

First, the novel frequency swapping technique was incorporated into the quasi-peak (QP) amplitude and average (AVG) amplitude parameters with $\pm 9 \%$ frequency swapping range, and the performance of this system was compared with another system without the technique. Because the two windings of a common mode choke are connected in series to two output lines, when the output currents flow through each winding, the magnetic flux direction formed in the iron core is inverse and can be neutralized. The magnetic flux in the iron core at balance is zero. Thus, the change in common mode inductance must consider the saturation from the increasing input current. Accordingly, the common mode choke (choke $=20 \mathrm{mH}$ ) was set as a fixed value to avoid other factors from causing spurious comparisons of the conducted EMI. In the following comparison, the X-capacitor capacitance is the only value that is adjusted in order to observe whether the $X$-capacitor capacitance amplitudes can be further decreased with the frequency swapping range of $\pm 9 \%$, and the EN55022 standards is still complied with in the meantime.

For the EMI measurement, the spectral range of $150 \mathrm{kHz}-30 \mathrm{MHz}$ was observed. In Figure 20a, no frequency swapping signals were added to the neutral end measurement. The QP amplitude testing value corresponding to the EN55022 standard satisfied the QP margin of more than $6 \mathrm{~dB} \mu \mathrm{V}$. In comparison, the AVG amplitude testing value corresponding to the EN55022 standard yielded an average margin of less than $6 \mathrm{dB \mu V}$ at 428, 498, and $1499 \mathrm{kHz}$. The lowest readings were 4.28, -2.79 , and $3.59 \mathrm{~dB} \mu \mathrm{V}$, respectively. Thus, EMI shielding components, including an augmented common mode choke, $X$-capacitor, and $Y 1$-capacitor, must be added to the system to improve the AVG amplitude.

Figure 20b shows that the measurement at the line end without the frequency swapping signals attains a QP amplitude that corresponds to the EN55022 standard and satisfies a QP margin of more than $6 \mathrm{~dB} \mu \mathrm{V}$. By contrast, the AVG amplitude corresponding to the EN55022 standard does not exceed the AVG margin of more than $6 \mathrm{dB \mu V}$ at 498 and $3867 \mathrm{kHz}$, yielding the lowest readings at -0.25 and $0.23 \mathrm{~dB} \mu \mathrm{V}$, respectively. Thus, systems must be equipped with EMI shielding components to improve the AVG amplitude.

How to set the frequency swapping range is described in this passage. The FB pin impedance $\left(R_{\text {total }}\right)$ is changed from $8.61 \mathrm{k} \Omega$ to $40 \mathrm{k} \Omega$. Figure 21a shows that in the neutral end measurement with the $\pm 9 \%$ frequency swapping signals, the QP amplitude corresponding to the EN55022 standard satisfies the QP margin of more than $6 \mathrm{~dB} \mu \mathrm{V}$. By comparison, the AVG amplitude corresponding to the EN55022 standard satisfies the QP margin of more than $6 \mathrm{~dB} \mu \mathrm{V}$ in the range of $150 \mathrm{kHz}-30 \mathrm{MHz}$. When all other system components were identical, adjusting the frequency swapping signals substantially improved QP and AVG amplitude. Moreover, the EMI shielding components did not require reinforcement. Compared with Figure 20a, the AVG amplitude at approximately $498 \mathrm{kHz}$ was improved by $13.24 \mathrm{~dB} \mu \mathrm{V}$, which was the largest improvement. In addition, the AVG amplitude at approximately $14.9 \mathrm{MHz}$ was improved by $13.18 \mathrm{~dB} \mu \mathrm{V}$. Figure $20 \mathrm{~b}$ shows that in line end measurement added with $\pm 9 \%$ frequency swapping signals, QP amplitude testing value corresponding to the EN55022 standard can satisfy the QP margin of higher than $6 \mathrm{~dB} \mu \mathrm{V}$. Compared with Figure 20b, this result shows that the AVG amplitude was improved by 12.65 and $11 \mathrm{~dB} \mu \mathrm{V}$ at approximately 498 and $3867 \mathrm{kHz}$, respectively. 


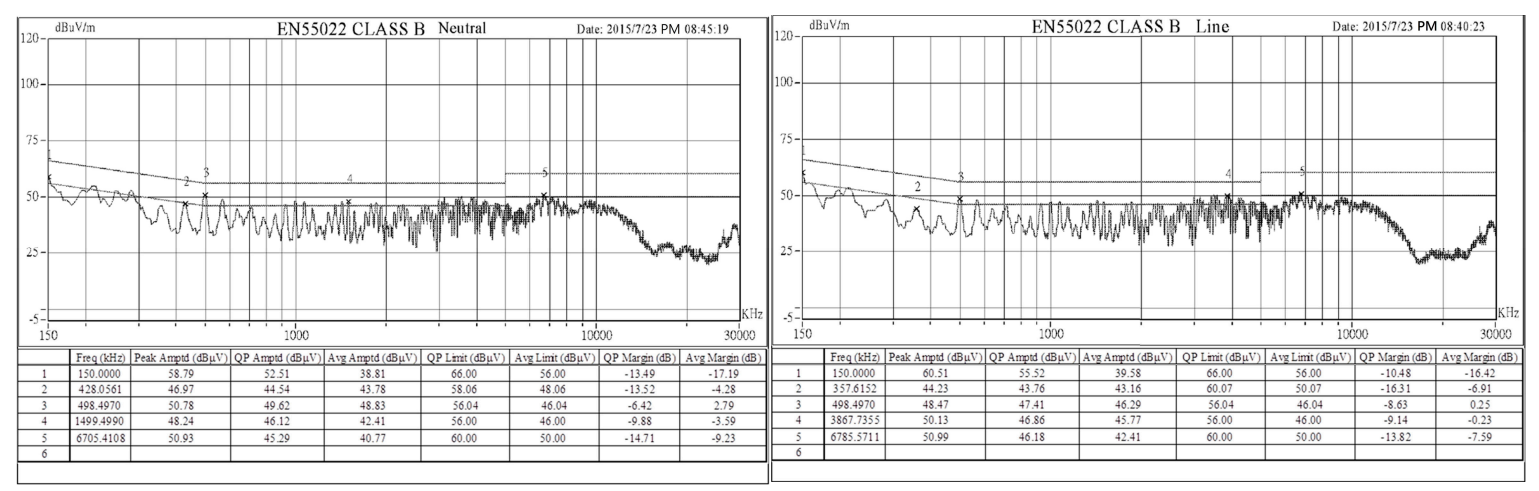

(a)

(b)

Figure 20. Conducted EMI measurements in the X-capacitor of $0.33 \mu \mathrm{F}$ (fixed frequency): (a) neutral; (b) line.

Comparison of the spectral amplitudes with and without frequency swapping shows that the harmonic amplitudes of switch voltage $\mathrm{Vds}$ were clearly suppressed with frequency swapping technique. The amplitudes from the fundamental wave to the 8th-order sub-harmonic wave decreased. For certain orders of harmonic waves (e.g., 2nd, 3rd, 5th and 7th orders), a maximum difference of $13.24 \mathrm{~dB}$ in the average amplitude was generated, showing that the novel frequency swapping technique is effective in suppressing conducted EMI.

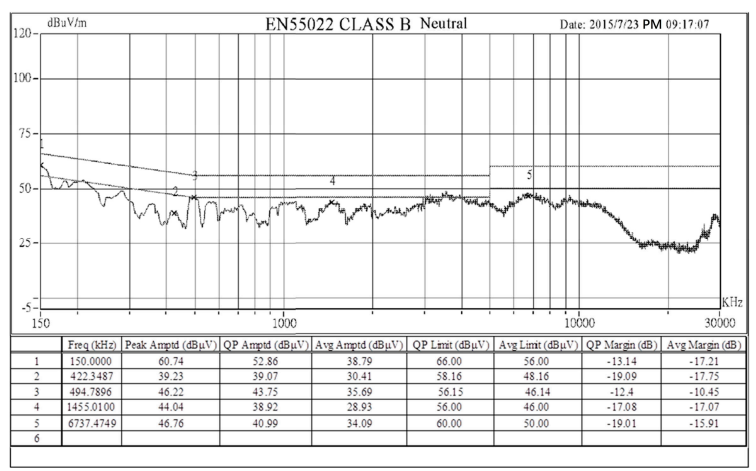

(a)

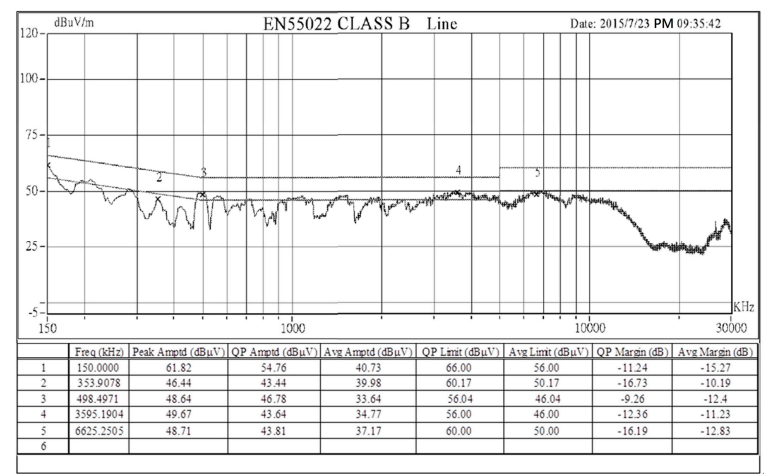

(b)

Figure 21. Conducted EMI measurements in the $X$-capacitor of $0.33 \mu \mathrm{F}$ ( $\pm 9 \%$ frequency swapping): (a) neutral; (b) line.

\section{Comparison of Conducted Electromagnetic Interference with Distinct X-Capacitor Values}

$X$-capacitors are a common capacitor in EMI filtration. Because $X$-capacitors are cross-connected to the line and neutral ends, $X$-capacitors do not have current leakage problems. The primary goal of $X$-capacitors is to suppress differential mode noise. Because the interference frequency is low, $X$-capacitors must be augmented to filter interference noise. Subsequent analysis involves identifying whether adding the novel frequency swapping technique on an original system can decrease the $X$-capacitor capacitance to conserve components and reduce layout area.

Figure 22a,b shows that in the measurements derived at the conduction EMI, the $X$-capacitor was reduced from 0.33 to $0.22 \mu \mathrm{F}$ (fixed frequency), which tells when the system is equipped with $0.22 \mu \mathrm{F}$ $X$-capacitor, the EMI-inhibition performance of $\pi$ filter becomes worse. Compared with systems with $0.33 \mu \mathrm{F} \mathrm{X}$-capacitor, the conduction EMI measurement raises up wholly in the range of $150 \mathrm{kHz}$ to $30 \mathrm{MHz}$. There is less margins for EN55022 standards. Hence, the proposed frequency swapping is involved in the system with $0.22 \mu \mathrm{F} \mathrm{X}$-capacitor to prove the improvements. 


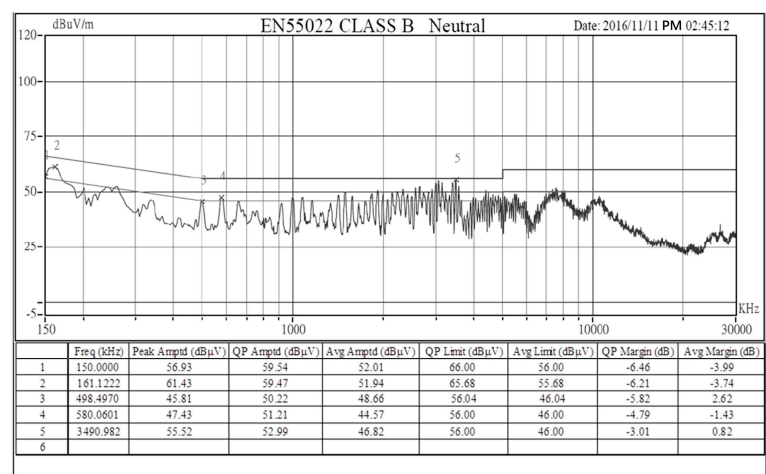

(a)

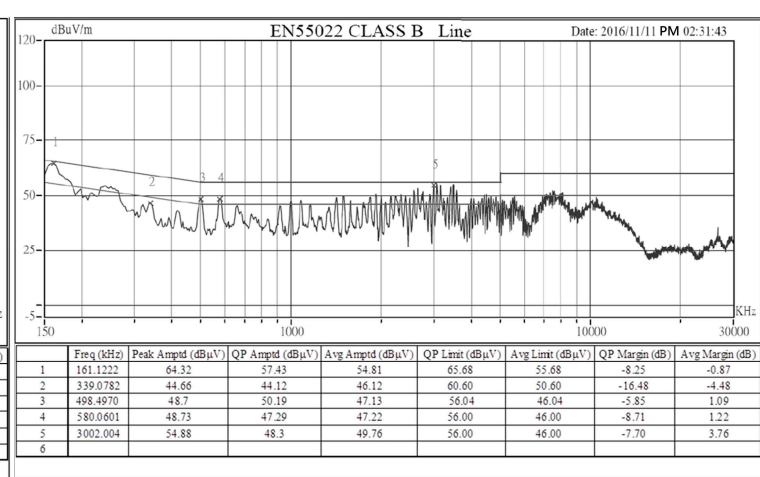

(b)

Figure 22. Conducted EMI measurements in the $X$-capacitor of $0.22 \mu \mathrm{F}$ (fixed frequency): (a) neutral; (b) line.

Figure 23a shows that in the measurements derived at the neutral end, the X-capacitor was reduced from 0.33 to $0.22 \mu \mathrm{F}$ ( $\pm 9 \%$ frequency swapping), and the QP amplitude corresponding to the EN55022 standard can satisfy the QP margin of more than $6 \mathrm{~dB} \mu \mathrm{V}$. However, the filtration frequency declined 0.5-fold. At $150 \mathrm{kHz}$, the QP amplitude was decreased by $1.93 \mathrm{~dB} \mu \mathrm{V}$, indicating that decreasing the X-capacitor affects the QP amplitude. In addition, the AVG amplitude corresponding to the EN55022 standard can satisfy the QP margin of more than $6 \mathrm{~dB} \mu \mathrm{V}$ in the range of $150 \mathrm{kHz}-30 \mathrm{MHz}$.

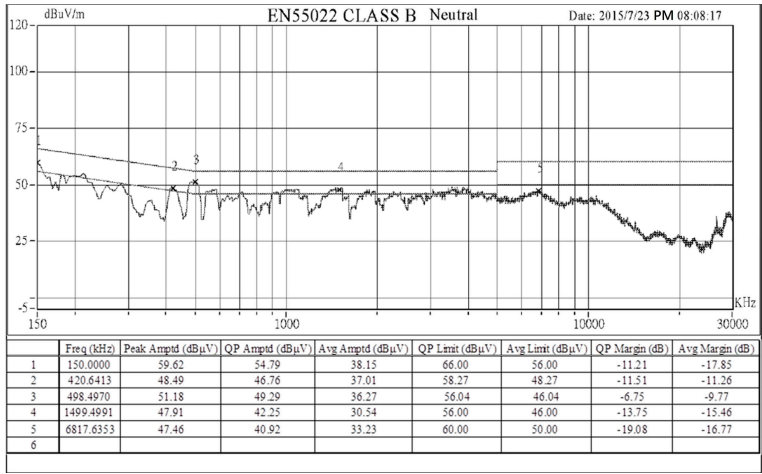

(a)

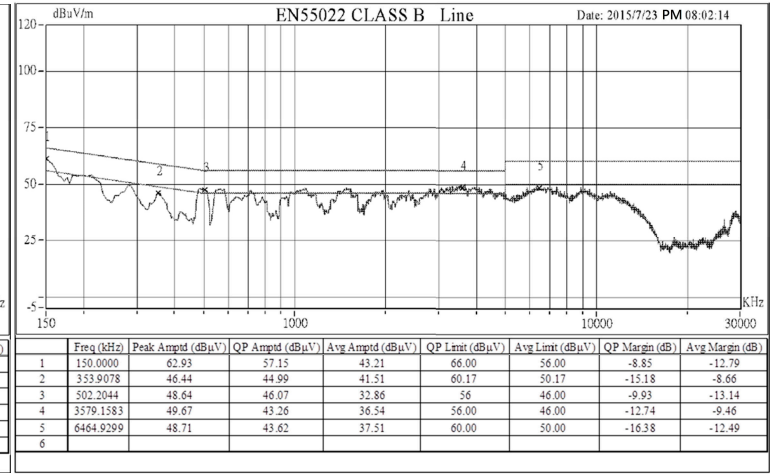

(b)

Figure 23. Conducted EMI measurements in the $X$-capacitor of $0.22 \mu \mathrm{F}$ ( $\pm 9 \%$ frequency swapping): (a) neutral; (b) line.

The lowest margin is $9.77 \mathrm{~dB} \mu \mathrm{V}$ at $498 \mathrm{kHz}$, which still conforms to EN55022 standards. Figure 23b reveals that in the line end measurement, the $X$-capacitor is decreased from 0.33 to $0.22 \mu \mathrm{F}$, and the $\mathrm{QP}$ amplitude corresponding to the EN55022 standard can satisfy the QP margin of more than $6 \mathrm{~dB} \mu \mathrm{V}$. Similarly, the filtration frequency is decreased by 0.5 -fold. At $150 \mathrm{kHz}$, the QP amplitude is decreased by $2.39 \mathrm{~dB} \mu \mathrm{V}$. The testing results show that decreasing the $X$-capacitor affects the QP amplitude. Moreover, the average amplitude corresponding to the EN55022 standard can satisfy the QP margin of more than $6 \mathrm{~dB} \mu \mathrm{V}$ in the range of $150 \mathrm{kHz}$ to $30 \mathrm{MHz}$. The lowest margin is $8.66 \mathrm{~dB} \mu \mathrm{V}$ at $353 \mathrm{kHz}$, which still conforms to EN55022 standards. Thus, the X-capacitor of the system $\pi$ filter can be decreased to $0.22 \mu \mathrm{F}$.

In response to the LC filter order, the first-order LC filter owns the capability of $40 \mathrm{~dB}$ decay. EMI refers to the frequency to attenuate a maximum conducted common mode noise measurement. In $12 \mathrm{~V} / 24 \mathrm{~W}$ applications, adding the novel frequency swapping technique can reduce the X-capacitor to $0.22 \mu \mathrm{F}$. Experimental results show Table 4 that the conducted EMI at both the neutral and line ends 
can pass the EN55022 standard; in addition, both the QP and AVG amplitude margins are more than $6 \mathrm{~dB} \mu \mathrm{V}$.

Table 4. Comparison of conducted EMI measurements. QP: Quasi-peak.

\begin{tabular}{|c|c|c|c|c|c|c|}
\hline \multirow{2}{*}{ Items } & & Frequency & QP Limit & Average Limit & QP Margin & Average Margin \\
\hline & & $\mathbf{k H z}$ & $\mathrm{dB} \mu \mathrm{V}$ & $\mathrm{dB} \mu \mathrm{V}$ & $\mathrm{dB}$ & $\mathrm{dB}$ \\
\hline \multirow{4}{*}{$\begin{array}{l}X \text {-capacitor of } 0.33 \mu \mathrm{F} \\
\quad \text { (fixed frequency) }\end{array}$} & \multirow{2}{*}{ Neutral } & 428.0561 & 58.06 & 48.06 & -13.52 & -4.28 \\
\hline & & 498.4970 & 56.04 & 46.04 & -6.42 & 2.79 \\
\hline & \multirow{2}{*}{ Line } & 357.6152 & 60.07 & 50.07 & -16.31 & -6.91 \\
\hline & & 498.497 & 56.04 & 46.04 & -8.63 & 0.25 \\
\hline \multirow{4}{*}{$\begin{array}{c}X \text {-capacitor of } 0.33 \mu \mathrm{F} \\
\text { ( } \pm 9 \% \text { frequency } \\
\text { swapping })\end{array}$} & \multirow{2}{*}{ Neutral } & 422.3487 & 58.16 & 48.16 & -19.09 & -17.75 \\
\hline & & 494.7896 & 56.15 & 46.14 & -12.4 & -10.45 \\
\hline & \multirow{2}{*}{ Line } & 353.9078 & 60.17 & 50.17 & -16.73 & -10.19 \\
\hline & & 498.4971 & 56.04 & 46.04 & -9.26 & -12.4 \\
\hline \multirow{4}{*}{$\begin{array}{l}X \text {-capacitor of } 0.22 \mu \mathrm{F} \\
\text { (fixed frequency) }\end{array}$} & \multirow{2}{*}{ Neutral } & 498.4970 & 45.81 & 50.22 & -5.82 & 2.62 \\
\hline & & 580.0601 & 47.43 & 51.21 & -4.79 & -1.43 \\
\hline & \multirow[b]{2}{*}{ Line } & 339.0782 & 44.66 & 44.12 & -16.48 & -4.48 \\
\hline & & 498.4970 & 48.7 & 50.19 & -5.85 & 1.09 \\
\hline \multirow{4}{*}{$\begin{array}{c}X \text {-capacitor of } 0.22 \mu \mathrm{F} \\
\text { ( } \pm 9 \% \text { frequency } \\
\text { swapping) }\end{array}$} & \multirow[b]{2}{*}{ Neutral } & 420.6413 & 58.27 & 48.27 & -11.51 & -11.26 \\
\hline & & 498.497 & 56.04 & 46.04 & -6.75 & -9.77 \\
\hline & \multirow{2}{*}{ Line } & 353.9078 & 60.17 & 50.17 & -15.18 & -8.66 \\
\hline & & 502.2044 & 56 & 46 & -9.93 & -13.14 \\
\hline
\end{tabular}

\section{Conclusions}

This paper explored the effect of frequency swapping techniques on suppressing EMI in a converter. FM PWM wave spectral characteristics were analyzed in detail. The spectra of the modulated waveform were calculated. A novel adjustable QRF converter frequency swapping method was proposed. Through analog modulation circuits and ICs, a frequency swapping function was implemented. In addition, testing of the QRF converter was conducted to verify the proposed technique. The proposed novel frequency swapping technique was verified experimentally. EMI testing revealed that the average $(150 \mathrm{kHz}-10 \mathrm{MHz})$ margin was attenuated by approximately $13.24 \mathrm{~dB} \mu \mathrm{V}$, effectively reducing the EMI caused by the converter. The conclusions of this paper are summarized as follows:

(1) The novel frequency swapping technique can effectively reduce the EMI caused by the converters. EMI testing showed that the average $(150 \mathrm{kHz}$ to $10 \mathrm{MHz})$ margin was attenuated by approximately $13.24 \mathrm{~dB} \mu \mathrm{V}$.

(2) The spectral distribution of the modulation wave is related to the slope of the modulation ratio. A well-performed spectral distribution is obtained by setting the swapping range to $\pm 9 \%$. When the spectrum of the modulation wave is non-modulated, there is no swapping frequency mechanism and vice versa. This pattern could be used to predict the spectral structures for the modulation waves of other shapes.

(3) When close-loop oscillation and oscillation noise factors are considered, the switching frequency offset and modulation frequency should not be excessive. In the selection of the modulation parameters, the frequency offset must be determined first. The offset adopted in this experiment was approximately $9 \%$, which caused zero abnormality in the original circuit.

(4) Applying the novel frequency swapping technique can reduce the $X$-capacitor in the system $\pi$ filter to $0.22 \mu \mathrm{F}$. The EN55022 standard was met regardless of testing the conducted EMI at the neutral or line ends. Both the QP and AVG amplitude margins were more than $6 \mathrm{~dB} \mu \mathrm{V}$. Thus, the proposed technique has the advantages of conserving the system components and reducing the layout area. 
Acknowledgments: This work was supported by the National Science Council of Taiwan under Contract Ministry of Science and Technology (MOST) 104-2221-E-011-077. The authors would like to thank Leadtrend Technology Corp. for the support on chip fabrication, measurement, and verifications.

Author Contributions: Both of the authors played important roles during the design of the novel frequency swapping technique and partially wrote the paper. Ming-Chang Tsou ran the simulation and did the experiment and Ming-Tse Kuo reviewed and revised the manuscript.

Conflicts of Interest: The authors declare no conflict of interest.

\section{References}

1. Wang, Y.-F.; Yang, L.; Wang, C.-S.; Li, W.; Qie, W.; Tu, S.-J. High step-up 3-phase rectifier with fly-back cells and switched capacitors for small-scaled wind generation systems. Energies 2015, 8, 2742-2768. [CrossRef]

2. Arango, E.; Ramos-Paja, C.A.; Calvente, J.; Giral, R.; Serna, S. Asymmetrical interleaved DC/DC switching converters for photovoltaic and fuel cell applications-Part 1: Circuit generation, analysis and design. Energies 2012, 5, 4590-4623. [CrossRef]

3. Karveis, G.A.; Manias, S.N. Analysis and design of a flyback zero-current switched (ZCS) quasi-resonant (QR) AC/DC converter. In Proceedings of the 27th Annual IEEE Power Electronics Specialists Conference, Baveno, Italy, 23-27 June 1996.

4. Hsieh, P.C.; Chang, C.J.; Chen, C.L. A primary-side-control quasi-resonant flyback converter with tight output voltage regulation and self-calibrated valley switching. In Proceedings of the 2013 IEEE Energy Conversion Congress and Exposition, Denver, CO, USA, 15-19 September 2013.

5. Wang, Z.; Wu, X.; Chen, M.; Zhang, J. Optimal design methodology for the current-sharing transformer in a quasi-resonant $(\mathrm{QR})$ flyback LED driver. In Proceedings of the Twenty-Seventh Annual IEEE Applied Power Electronics Conference and Exposition, Orlando, FL, USA, 5-9 February 2012.

6. Kuo, M.-T.; Tsou, M.-C. Simulation of standby efficiency improvement for a line level control resonant converter based on solar power systems. Energies 2015, 8, 338-355. [CrossRef]

7. EN 55022, CISPR 22. Available online: http://www.rfemcdevelopment.eu/en/en-55022-2010 (accessed on 22 December 2016).

8. González, D.; Gago, J.; Balcells, J. New simplified method for the simulation of conducted EMI generated by switched power converters. IEEE Trans. Ind. Electron. 2003, 50, 1078-1084. [CrossRef]

9. Santolaria, A.; Balcells, J.; González, D. Theoretical and experimental results of power converter frequency modulation. In Proceedings of the IECON 02 IEEE 2002 28th Annual Conference of the Industrial Electronics Society, Seville, Spain, 5-8 November 2002.

10. Lin, F.; Chen, D.Y. Reduction of power supply EMI emission by switching frequency modulation. IEEE Trans. Power Electron. 1994, 9, 132-137.

11. Paul, C.R. Transmission Lines in Digital Systems for EMC Practitioners; Wiley: Hoboken, NJ, USA, 2012.

12. Zhang, D.; Chen, D.; Sable, D. Non-intrinsic differential mode noise caused by ground current in an off-line power supply. In Proceedings of the 29th Annual IEEE Power Electronics Specialists Conference, PESC 98 Record, Fukuoka, Japan, 22-22 May 1998; pp. 1131-1133.

13. Kchikach, M.; Yuan, Y.S.; Qian, Z.M.; Pong, M.H. Simple modeling for conducted common-mode current in switching circuits. In Proceedings of the PESC Record-IEEE 32nd Annual Power Electronics Specialists Conference, Vancouver, BC, Canada, 17-22 June 2001.

14. Kuisma, M. Variable frequency switching in power supply EMI-control: An overview. IEEE Aerosp. Electron. Syst. Mag. 2003, 18, 18-22. [CrossRef]

15. Maillet, Y.; Lai, R.; Wang, S.; Wang, F.; Burgos, R.; Boroyevich, D. High-density EMI filter design for dc-fed motor drives. IEEE Trans. Power Electron. 2010, 25, 1163-1172. [CrossRef]

16. Ala, G.; Giaconia, G.C.; Giglia, G.; Di Piazza, M.C.; Luna, M.; Vitale, G.; Zanchetta, P. Computer aided optimal design of high power density EMI filters. In Proceedings of the 2016 IEEE 16th International Conference on Environment and Electrical Engineering, Florence, Italy, 6-8 June 2016.

17. Ala, G.; Di Piazza, M.C.; Giaconia, G.C.; Giglia, G.; Vitale, G. Design and performance evaluation of a high power-density emi filter for PWM inverter-fed induction-motor drives. IEEE Trans. Ind. Appl. 2016, 52, 2397-2404. [CrossRef] 
18. Touré, B.; Schanen, J.L.; Gerbaud, L.; Meynard, T.; Roudet, J.; Ruelland, R. EMC modeling of drives for aircraft applications: Modeling process, EMI filter optimization, and technological choice. IEEE Trans. Power Electron. 2013, 28, 1145-1156. [CrossRef]

19. Hardin, K.B.; Fessler, J.T.; Bush, D.R. A study of the interference potential of spread spectrum clock generation techniques. In Proceedings of the IEEE International Symposium on Electromagnetic Compatibility, Atlanta, GA, USA, 14-18 August 1995.

20. TOP242-TOP250 Reference Design. Available online: http://chinese.powerint.com/dak.zh-cn.html (accessed on 8 March 2016).

21. LTC6902 Datasheet. Available online: http://datasheet.eeworld.com.cn/pdf/28512_LINER_LTC6902.html (accessed on 9 August 2016).

22. NCP1215A Datasheet. Available online: http://datasheet.eeworld.com.cn/pdf/12824_ONSEMI_NCP1215. html (accessed on 9 August 2016).

23. ICE3B0365J Datasheet. Available online: http://datasheet.eeworld.com.cn/pdf/151232_INFINEON_ ICE3B0365J.html (accessed on 9 August 2016).

24. FSDH0265 Series Datasheet. Available online: http://datasheet.eeworld.com.cn/pdf/37029_FAIRCHILD_ FSDH0265RL.html (accessed on 9 August 2016).

25. Sendra, J.B.I. SSCG Methods of EMI Emission Reduction applied to Switching Power Converters. Ph.D. Thesis, BarcelonaTech, Barcelona, Spain, 2004.

(C) 2016 by the authors; licensee MDPI, Basel, Switzerland. This article is an open access article distributed under the terms and conditions of the Creative Commons Attribution (CC-BY) license (http://creativecommons.org/licenses/by/4.0/). 